\title{
Activités
}

\section{La conceptualisation et la place des concepts pragmatiques dans l'activité professionnelle et le développement des compétences}

Conceptualisation, pragmatical concepts in professional activity and competence development

La conceptualización y el lugar de los conceptos pragmáticos en la actividad profesional y en el desarrollo de las competencias

\section{Christine Vidal-Gomel et Janine Rogalski}

\section{OpenEdition}

Journals

Édition électronique

URL : https://journals.openedition.org/activites/1401

DOI : 10.4000/activites. 1401

ISSN : $1765-2723$

Éditeur

ARPACT - Association Recherches et Pratiques sur les ACTivités

Référence électronique

Christine Vidal-Gomel et Janine Rogalski, « La conceptualisation et la place des concepts

pragmatiques dans l'activité professionnelle et le développement des compétences ", Activités [En ligne], 4-1 | avril 2007, mis en ligne le 15 avril 2007, consulté le 28 juin 2022. URL : http:// journals.openedition.org/activites/1401; DOI : https://doi.org/10.4000/activites.1401

\section{(C) $\odot \Theta \Theta$}

Activités est mis à disposition selon les termes de la licence Creative Commons Attribution - Pas d'Utilisation Commerciale - Pas de Modification 4.0 International. 


\title{
La conceptualisation et la place des concepts pragmatiques dans l'activité professionnelle et le développement des compétences
}

\author{
Christine Vidal-Gomel \\ Laboratoire « Paragraphe » (EA 349), équipe « Ethnométhodologie \& Cognition » \\ Université Paris 8 - UFR de psychologie \\ 2, rue de la liberté - 93526 Saint-Denis Cedex 2 - christine.vidal@univ-paris8.fr \\ Janine Rogalski \\ Laboratoire « Cognition \& Usages » (EA 4004) \\ Université Paris 8 - UFR de psychologie \\ 2, rue de la liberté - 93526 Saint-Denis Cedex 2 - Janine.rogalski@univ-paris8.fr
}

\begin{abstract}
Conceptualisation, pragmatical concepts in professional activity and competence development. The aim of the paper is to analyse the place of conceptualisation in professional activities, and to specify the notion of « pragmatical concept » (Samurçay, \& Pastré, 1995), which plays a key role in professional didactics, as a component of professional competence and as an organiser of the activity at work. The history of the notion is briefly presented, and the various approaches - directly or indirectly dealing with the issue of conceptualisation for action are discussed. Then, the paper develops the relationships and differences between - on the one hand - pragmatical concepts and the concepts of science and technics, and - on the other hand - everyday or "spontaneous » concepts. We discuss then the theoretical place respectively played by pragmatical concepts in professional didactics and « concepts-in-action » in disciplinary didactics (mathematics: Vergnaud, 1990). Finally, we propose some examples of how the issue of conceptualisation for action may be used in the design of operative cognitive tools, which are specific type of tools for supporting professional work.
\end{abstract}

KEYWORDS

conceptualisation, development of professional competence, pragmatical concept, operative cognitive tools.

\section{Introduction}

Le questionnement sur la conceptualisation dans l'activité professionnelle s'est développé dans différentes approches, avec des terminologies variées exprimant des notions ${ }^{1}$ liées à des cadres théoriques de référence, eux-mêmes divers. Schématiquement, en ergonomie cognitive, les recherches sur l'expertise ont étudié l'organisation des connaissances; les études de l'activité en environnement dynamique ont particulièrement insisté sur le rôle des modèles mentaux et sur les différences de systèmes de représentation entre débutants et experts. Toutefois, bien que la nature de la conceptualisation ait pu être placée au cœur de l'étude de l'émergence et du développement de l'expertise, ce sont essentiellement les approches développementales chez l'enfant et l'élève qui ont mis en avant l'importance des processus de conceptualisation et le rôle des changements conceptuels dans différents

1. En toute rigueur nous devrions utiliser le terme de « concepts », puisqu'il s'agit de concepts théoriques, mais nous utiliserons aussi le terme de « notion » pour éviter des expressions du type « concept de concept...». 
domaines de savoir. La didactique professionnelle se situe dans cette lignée : elle a souligné l'importance de la conceptualisation, ne se réduisant pas au traitement de l'information (Rogalski, 2004), et a mis en évidence l'existence d'un type de concepts distincts des concepts scientifiques et techniques, comme des concepts quotidiens : les concepts pragmatiques (Samurçay, \& Pastré, 1995).

Cet article vise à faire le point sur cette notion, et la situer par rapport à d'autres types de concepts, sans effacer la variété de terminologies qui ont été ou sont toujours utilisées. Nous reviendrons dans un premier temps sur les travaux à l'origine de la notion de concept pragmatique, et nous en présenterons plusieurs exemples, pour illustrer notre propos. S'intéresser aux concepts pragmatiques nécessite d'aborder la conceptualisation pour l'action : nous situerons cette question au regard de la littérature, sans prétendre à une revue exhaustive hors de portée dans un article. Nous développerons ensuite comment les concepts pragmatiques se différencient d'autres types de concepts avec, à un pôle, les concepts scientifiques et techniques et, à un autre pôle, les concepts spontanés et quotidiens (Vygotsky, 1934/1997). Enfin, la filiation historique entre la notion de concept pragmatique et celles de concept-en-acte et de théorème-en-acte (Vergnaud, 1990) conduit à s'interroger sur leurs différences, et à situer leur place dans des modèles de développement de la conceptualisation. Pour conclure, nous discuterons de l'intérêt de la prise en compte en ergonomie de la conceptualisation et des concepts pragmatiques dans la conception des systèmes d'aide particuliers que sont les outils cognitifs opératifs (Rogalski, \& Samurçay, 1993 ; Rogalski, \& Durey, 2004).

\section{1.- Les concepts pragmatiques}

\section{1.- L'émergence de la notion de concept pragmatique}

La notion de concept pragmatique est issue de la convergence de recherches réalisées dans différents domaines professionnels. Ces recherches avaient conduit à identifier des « entités » qui structuraient l'activité efficace des opérateurs et qui n'étaient ni des paramètres directement observables ou mesurés via des instruments, ni des concepts scientifiques ou techniques.

La confrontation de ces recherches dans le cadre de la didactique professionnelle a mis en évidence une variété de concepts pragmatiques : ainsi « bourrage » dans la plasturgie, «état thermique » dans la conduite de haut-fourneau, « espaces menacés possibles» au rugby, «axe de propagation » et « front» du feu dans la lutte contre les feux de forêt, sont cités comme concepts pragmatiques (GR « didactique professionnelle », 19952). Les dénominations de ces «entités » ont, dans un premier temps, varié : « variables intermédiaires » (Samurçay, \& Hoc, 1988), « descripteurs » (Hoc, 1989), « variables tactiques » et « variables construites » (Samurçay, \& Rogalski, 1992) ou « concepts-enacte » (Pastré, 1992a).

Ainsi, les premières publications de Pastré (1992a) parlent du bourrage comme d'un concept-enacte, le rattachant ainsi au cadre théorique de Vergnaud (1990). La notion de bourrage sera plus tard reconnue comme un concept pragmatique central dans la plasturgie (Pastré, 2004a, par exemple), et utilisée comme exemple paradigmatique.

Concernant la conduite de hauts-fourneaux, et plus particulièrement les activités de diagnostic, Samurçay et Hoc (1988) ont d'abord parlé de variables intermédiaires, pour les différencier des variables d'entrée et de sortie, puis de descripteurs globaux par opposition à des paramètres isolés. Dans un article ultérieur, Hoc précise que les descripteurs ne sont pas accessibles directement et permettent d'inférer des phénomènes internes au haut-fourneau à partir de paramètres accessibles (Hoc, 1989).

Rapprochant la notion de descripteur, élaborée pour rendre compte de l'activité de conduite des hauts-fourneaux, et celle de variable tactique, utilisée pour rendre compte de l'activité de lutte contre les feux de forêt, Samurçay et Rogalski (1992) définissent la notion plus générale de variable construite.

2. Document interne au Groupe de Recherche. 
Enfin, c'est dans un texte de 1995, intitulé « la conceptualisation des situations de travail dans la formation des compétences », que Samurçay et Pastré synthétisent ces différents apports et stabilisent le terme de concept pragmatique. On peut reprendre pour ce terme la caractérisation proposée par Samurçay et Rogalski (1992, p. 235) : « représentations schématiques et opératives, élaborées par et pour l'action, qui sont le produit d'un processus historique et collectif, et qui sont transmises essentiellement par expérience et par compagnonnage ».

Les représentations dont il est ici question sont des représentations pour l'action : « réseaux de propriétés, de concepts, de savoirs, de savoir-faire, de croyances, de sensations éprouvées, construites, sélectionnées au cours de l'histoire du sujet, à partir de sa formation, de son expérience, et des besoins de l'action » (Weill-Fassina, Rabardel, \& Dubois, 1993, p. 17).

L'exemple paradigmatique de concept pragmatique est celui du bourrage identifié et analysé par Pastré dans le domaine de la plasturgie (1992a, 1994, 1997, 2004a). Le bourrage désigne un état d'équilibre ou de déséquilibre entre deux pressions, celle qui est exercée par la machine et celle qui est exercée par la matière, au moment où on passe de la phase d'injection de la matière dans le moule, à celle où l'on maintient la matière dans le moule (cette transition est appelée " commutation »). Un bon réglage de la machine consiste à effectuer la commutation au moment où le moule est bien plein, pour éviter les défauts de fabrication. Les opérateurs compétents disposent d'un ensemble d'indicateurs, et notamment le mouvement de la machine après commutation, pour évaluer le bourrage ; cela leur permet aussi d'identifier le régime de fonctionnement de la machine et de réaliser des réglages afin d'anticiper les défauts de fabrication. Ce concept pragmatique est propre à la situation de plasturgie ; il appartient au monde de la conduite et du réglage de la machine ; il est étroitement lié au fonctionnement de la machine. Bien qu'on puisse le définir en relation avec la notion physique de pression, il s'agit d'un concept qualitatif et non d'un paramètre pour lequel il y aurait un point de mesure, ou une méthode de calcul.

\section{2.-Concepts pragmatiques : des invariants conceptuels et opératoires}

Les concepts pragmatiques ont une double face, l'une est tournée vers l'action en temps réel, l'autre vers l'organisation des représentations (Samurçay, \& Pastré, 1995 ; Pastré, 1999b) :

- ce sont des invariants opératoires qui structurent l'activité en permettant un couplage fort entre prise d'information et opérations exécutées ;

- ce sont des invariants conceptuels qui permettent aux représentations « de se structurer en mettant l'accent sur les relations essentielles existant entre les variables de la situation » (Samurçay, \& Pastré, 1995, p. 16).

\subsection{1.- Vers l'action en temps réel}

Les concepts pragmatiques constituent essentiellement une conceptualisation de l'objet de l'action, c'est le cas, par exemple, des descripteurs identifiés par Samurçay et Hoc (Hoc, 1989 ; Hoc, \& Samurçay, 1992 ; Samurçay, \& Hoc, 1988, 1996). Ils guident alors la prise d'information sur les paramètres observables ou mesurables pour la construction d'une représentation de la situation en cours (représentation occurrente).

Ils peuvent aussi représenter des moyens de l'action. Dans le cas de la lutte contre les feux de forêts, les concepts pragmatiques de vitesse, d'axe de propagation et de front de feu sont des représentations opératives du feu. Un autre concept pragmatique, plus général dans la gestion de sinistres, est celui de « balance des moyens », dont la valeur détermine les possibilités d'action : si les moyens disponibles sur le lieu de l'action au moment approprié sont supérieurs aux moyens nécessaires, l'action est possible (même si son succès n'est pas assuré).

Les deux concepts pragmatiques d'axe de propagation du feu et de vitesse du feu réduisent drastiquement la complexité de l'évolution du feu — tenant à la multiplicité des paramètres de distribu- 
tion de végétation sur le terrain, de variations topographiques locales, de mouvements aérologiques et de température tenant au vent et au phénomène même du feu. Ils permettent un « raisonnement tactique » d'anticipation globale de la menace, de besoins d'actions de protection, et de calcul des moments et lieux où l'action est possible pour arrêter la progression du feu (ou au moins la limiter); raisonnement suffisamment rapide pour que la décision à laquelle ils conduisent soit exécutable.

Ces concepts pragmatiques, de même que les « descripteurs » de l'état du haut-fourneau (état thermique, descente des charges, etc.), ont un caractère global permettant, pour agir en temps contraint, de « penser » des phénomènes complexes dont l'observation ne peut être complète (le feu), ou qui ne sont pas observables (les températures dans le feu, les phénomènes à l'intérieur du haut-fourneau, les pressions exercées sur la matière dans le moule en plasturgie, etc.). Cette caractéristique des concepts pragmatiques les rapproche des « concepts de haut niveau » qui ont été identifiés en analysant l'activité d'opérateurs experts (Randel, Pugh, \& Reed, 1996). Ils permettent de se constituer une vision d'ensemble («big picture », Ibid.) comprenant les éléments principaux, de sorte que le sujet n'a pas besoin de mettre en œuvre un raisonnement lourd, comprenant de nombreux pas (« working smart », Ibid.).

On discutera plus loin de leurs rapports avec des concepts scientifiques, et de la possibilité que de tels concepts pragmatiques soient le prélude à de futurs concepts scientifiques, qui en « prouveraient » le bien-fondé théorique. Dans les cas cités ci-dessus, la dimension pragmatique de couplage entre prise d'information et action nous semble donner une nécessité à leur caractère d'invariant opératoire, rassemblant une multiplicité en une seule entité « cognitivement manipulable ».

\subsection{2.- Vers l'organisation des représentations}

La seconde face des concepts pragmatiques est celle de leur statut du point de vue des représentations. Les concepts pragmatiques ne sont pas isolés. Ils s'inscrivent dans un réseau de relations, qui intègre des concepts pragmatiques et les relie à des paramètres directement observables. Ce réseau de relations comporte aussi les opérations qui permettent de modifier l'état de l'objet pour atteindre le but fixé à l'action. La notion de « structure conceptuelle» de la situation réfère à un tel réseau de relations (Pastré, 2005a).

Par exemple, dans le cas des feux de forêt, le concept pragmatique de « ligne d'arrêt » est lié à ceux de « front de feu », « axe de propagation du feu », et «balance des moyens ». La ligne d'arrêt fait référence à l'action de blocage de la progression d'un feu. Elle se situe face au front de feu (transversalement à l'axe de propagation) et peut être efficace si la balance des moyens est positive ; pour la réaliser il faut déplacer des engins en nombre permettant un débit d'eau adéquat. Dans le cas des hauts-fourneaux, des opérations sur des paramètres accessibles (injection de charbon, température de fluides, etc.) permettent de modifier, par exemple, l'état thermique (descripteur - concept pragmatique) (Hoc, \& Samurçay, 1992).

\section{3.- La structure conceptuelle de la situation}

La structure conceptuelle de la situation est organisée autour de l'action à conduire et repose sur l'élaboration du diagnostic de la situation. Elle est composée de trois éléments principaux (Pastré, 2005a, p. 243) :

- « des concepts organisateurs de l'activité, qui sont les dimensions, extraites du réel dans sa globalité, qui vont permettre de fonder le diagnostic de la situation;

- des indicateurs ${ }^{3}$, qui sont des observables, naturels ou instrumentés, qui permettent pour une situation donnée, d'identifier la valeur que prennent les concepts organisateurs. L'équivalent d'une relation signifiant-signifié relie les indicateurs aux concepts ;

3. Les indicateurs sont toujours observables, mesurables, etc., ce qui n'est pas le cas des variables qui peuvent aussi être construites, abstraites (pas exemple la vigueur de la vigne). 
- des classes de situations, qui permettent d'orienter l'action en fonction du diagnostic effectué, et qui découlent des valeurs prises par les concepts organisateurs ».

Caens-Martin (2005) fournit un exemple de structure conceptuelle de la situation dans le domaine de la taille de la vigne (Figure 1).

À partir d'indicateurs - informations tactiles, comme la flexibilité des sarments, ou visuelles, comme le diamètre des sarments - les professionnels font des inférences sur ce que Caens-Martin (op. cit.) nomme des variables (qualité du bois, vigueur de la souche, architecture du cep, ou réseau de distribution de la sève). Sur cette base, les deux concepts organisateurs (la charge et l'équilibre) permettent de hiérarchiser les buts (la production ou la pérennité du système), d'élaborer un diagnostic de la situation (incluant le pronostic) et de choisir les règles d'action appropriées.

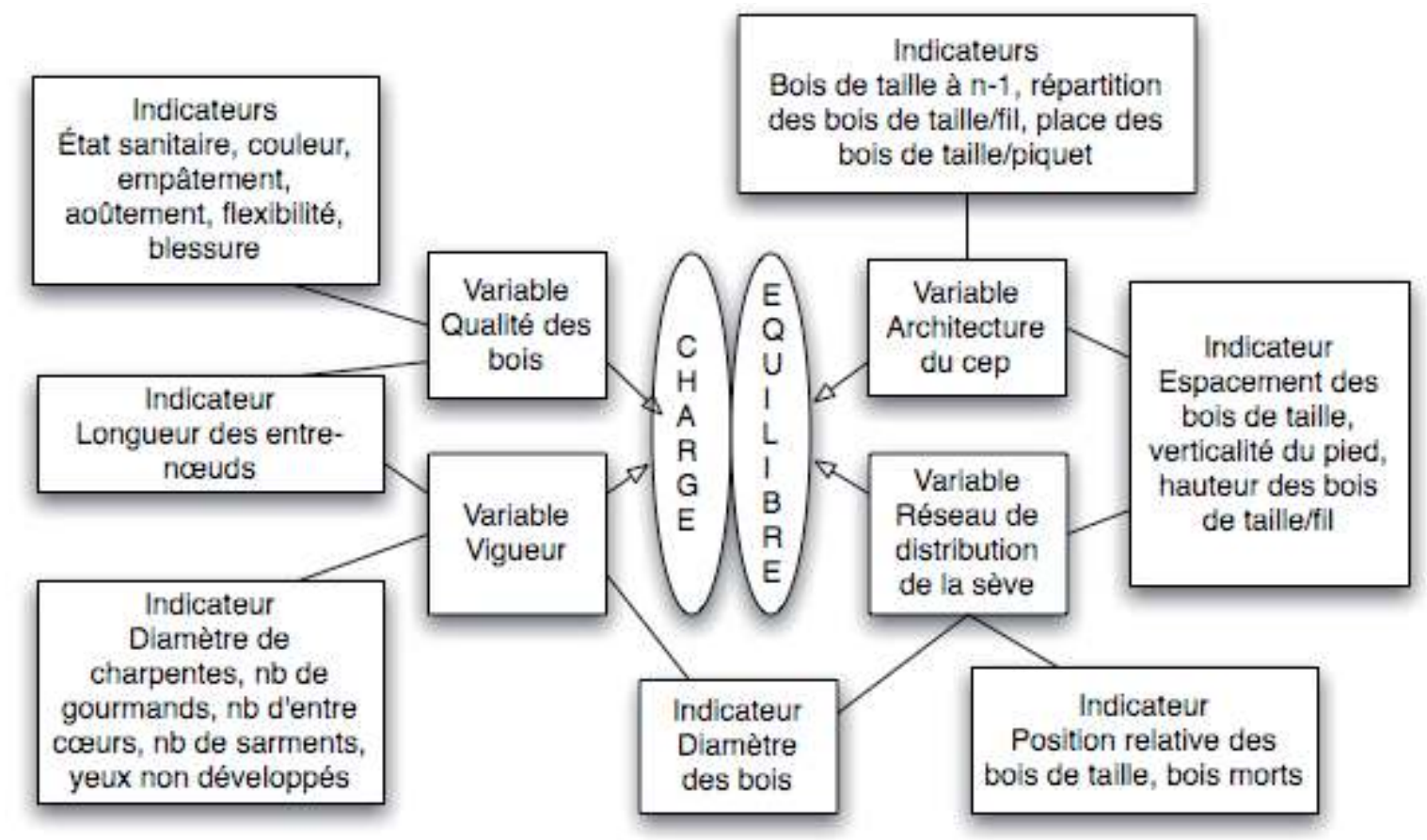

Figure 1 : La structure conceptuelle de la taille de la vigne (d'après CaensMartin, 2005).

On peut ici remarquer que les professionnels raisonnent avec un double niveau d'inférences et que chacun mobilise des concepts pragmatiques (figure 1). Les quatre variables identifiées (qualité du bois, etc.) pourraient en effet être considérées comme des concepts pragmatiques - dans son texte, l'auteur utilise d'ailleurs le terme de variables construites (op. cit., 2005, p. 88). Mais ce sont les concepts pragmatiques de charge et d'équilibre qui organisent l'activité - hiérarchisation des buts, diagnostic/pronostic de situation, et choix des règles d'action -, et donnent du sens à l'ensemble. Ce sont des organisateurs de l'activité au sens de Pastré (2005a) : ils sont inséparables de l'action efficace.

L'étendue du réseau de relations entre concepts pragmatiques et indicateurs dont disposent les opérateurs dépend de leur expérience et expertise, et de leur champ d'action. Ainsi pour les hauts-fourneaux, la structure conceptuelle exprimée et utilisée par les ingénieurs est plus étendue que celle des conducteurs (Samurçay, 1995).

On peut faire ici un bilan de l'utilisation des termes de variables, paramètres et indicateurs. Globalement les notions de paramètres et de variables sont assez proches. On utilise plutôt le terme de paramètre quand il s'agit d'une variable mesurable ou calculable. Il est le plus souvent un déterminant dans un réseau de relations entre variables alors qu'une variable peut être aussi bien un déterminé qu'un déterminant. Par ailleurs, on parle de « variable construite » ou de « variable hypothétique » et 
pas de paramètre. De même, on ne parle pas de « paramètre de sortie », mais de « variable de sortie ». La notion d'indicateur rend compte des informations qui sont sélectionnées dans le réel par l'opérateur. Dans une structure conceptuelle, les indicateurs sont inscrits dans une relation de signification avec des concepts pragmatiques, qu'ils permettent d'évaluer.

\section{2.- La conceptualisation dans l'activité et le développement des compétences}

La théorisation que propose la didactique professionnelle place les concepts pragmatiques au cœur des compétences et de leur développement (Samurçay \& Pastré, 1995, 2004 ; Rogalski, 2004 ; Pastré, Mayen \& Vergnaud, 2006). Nous considérons qu' "un élément essentiel dans le concept de compétence professionnelle est son rapport avec l'accomplissement de l'action dans une situation de travail $^{4} \gg$ (Rogalski \& Marquié, 2004, p. 142). Deux aspects des compétences peuvent être différenciés : « savoir ce qu'il faut faire » et « pouvoir le faire ». « Savoir que faire » rend compte du fait qu'il faut faire un diagnostic de la situation particulière à traiter, diagnostic qui peut rester implicite. « Pouvoir faire » renvoie à l'efficacité de l'action.

Compréhension et efficacité sont dans une relation complexe : savoir ce qu'il faut faire ne signifie pas pouvoir le faire et, réciproquement, l'action peut être réussie sans être comprise (Savoyant, 2004 ; Clausewitz, 1955). Piaget (1974) a différencié deux étapes de la coordination de l'action : la coordination agie de l'action et la coordination conceptuelle, étapes reprises dans l'analyse du développement des compétences ${ }^{5}$; la coordination conceptuelle étant comprise comme le moment « où la compréhension de l'action vient rattraper sa réussite, constituant ainsi un progrès décisif dans l'organisation de l'action » (Weill-Fassina \& Pastré, 2004, p. 217).

En nous intéressant aux concepts pragmatiques, nous nous intéressons au versant « savoir » des compétences, mais le terme «pragmatique » souligne que la conceptualisation est au service de l'action en cours, ce qui la distingue de la théorisation à visée épistémique. En effet, un « praticien est un opportuniste [... qui] ne cherche ni la complétude, ni la cohérence de son savoir, il cherche l'efficience » (Pastré, 1997, p. 94). Les concepts pragmatiques doivent être abordés dans ce cadre : ils sont le résultat d'un processus de développement de la conceptualisation dans et pour l'action.

Nous proposons un bref tour d'horizon de la place de la conceptualisation dans différentes approches, avant de situer comment se modifie le rapport entre concepts théoriques (scientifiques et techniques) et conceptualisation pragmatique dans le développement des compétences professionnelles.

\section{1.-La place de la conceptualisation dans différentes approches}

La conceptualisation a fait l'objet de très nombreuses recherches en sciences cognitives, comme le relève Kaufman (2001), ou dans les didactiques disciplinaires (Tiberghien, 2002). La majeure partie de ces travaux concerne des apprentissages disciplinaires et peu les apprentissages professionnels. Ce n'est pas sans lien avec le fait, qu'en psychologie, les études développementales ont largement porté sur les enfants et les adolescents et ont peu concerné le développement des adultes.

Dans les études et recherches sur les activités de travail, plusieurs approches peuvent aborder, plus ou moins directement, le rôle de la conceptualisation dans le développement des compétences professionnelles. Nous les différencions en précisant schématiquement leurs orientations principales.

\subsection{1.- En psychologie ergonomique, et en ergonomie}

La psychologie ergonomique, issue en partie de la psychologie cognitive, s'est intéressée surtout à

4. Pour plus de précisions sur les compétences professionnelles et leur développement voir Rogalski et Marquié (2004), Leplat et de Montmollin (2001), Pastré (1994).

5. Nous renvoyons à Pastré $(1994,1997)$ pour une analyse des apports de Piaget à la didactique professionnelle. 
l'apprentissage (transfert et « skill acquisition » y constituent des thématiques centrales), à l'organisation des connaissances, et à la caractérisation de l'expertise. Globalement, il s'agit de travaux qui se sont davantage centrés sur l'apprentissage que sur le développement, et la conceptualisation pour l'action n'est pas un thème traité en tant que tel. Certaines problématiques présentent toutefois une parenté avec celle de la conceptualisation pour l'action.

Ainsi, dans son étude de l'apprentissage, à côté du questionnement sur la représentation des connaissances, Patrick (1992) introduit les notions de " advance organisers », de " conceptual framework » et de «qualitative change ». Il souligne que « pour acquérir une nouvelle information, le sujet en formation doit avoir des concepts et des schémas appropriés, par lesquels il peut intégrer cette nouvelle information et lui donner du sens ${ }^{6} »$ (p. 319, notre traduction). Patrick donne l'exemple du « développement et raffinement d'un schéma, qui guide la perception d'une image radiologique par un expert ».

Restons dans les approches caractérisant «l'humain comme système de traitement de l'information ». Dans son ouvrage de psychologie cognitive intitulé « De l'interprétation de l'information à l'action », Richard (2004) différencie trois processus de conceptualisation : le recodage des propriétés à un niveau abstrait, la découverte de propriétés dans la construction du réseau sémantique associé à un domaine d'action, l'élaboration d'un système conceptuel permettant de justifier les procédures. La conceptualisation y est abordée sous l'angle de la catégorisation et, les relations entre les éléments d'un système qui sont examinées sont des relations d'inclusion, des relations " partie-tout » ou des relations d'état et de changement d'état entre des éléments d'un système ${ }^{7}$. Ce point de vue ne rend que partiellement compte de la conceptualisation. En effet, les relations au sein d'une structure conceptuelle de situation (figure 1, par exemple) peuvent être des relations multiples d'évolution et des relations de causalité (telles qu'on les trouve en physique dans un graphe de fluence). Par ailleurs, ces questions doivent être mises en rapport avec les débats portant sur la définition de ce qu'est un concept et ses liens avec la catégorisation.

Ainsi, Thagard et Toombs (2005) articulent explicitement la problématique de la catégorisation et celle du changement conceptuel - devenue classique en psychologie du développement (Carey, 1985 ; Vosniadou, \& Brewer, 1987). Ils présentent plusieurs approches des concepts - dont une approche qui les définit comme des prototypes et prend en compte les traits typiques -, et en retiennent "l'approche orientée par les connaissances» [ « knowledge approach», (Thagard, \& Toombs, op. cit., p. 245)], dans laquelle les concepts ont aussi un rôle crucial d'explication.

Ce point de vue est parent de celui de Richard (2004), qui aborde la conceptualisation en posant la question suivante : « les catégories ont-elles un contenu conceptuel ? ». Il y apporte une réponse positive : «on peut concilier la typicité (le degré de représentativité des exemplaires d'une catégorie) avec l'idée que les catégories ont un contenu conceptuel défini $»^{8}$ (p. 38). Ce type d'approche ne rend compte que partiellement des concepts pragmatiques. En effet, nombre de ces concepts, comme le front de feu, ne sont pas des catégories.

Dans leur chapitre, Thagard et Toombs développent également la notion de « concepts théoriques » qui renvoie à des « objets non observables », « des entités théoriques» (p. 244). Dans ce cadre, ils

6. Patrick (1992) introduit la notion de « conceptual framework» : organisation, structure de connaissances, incluant des buts, plans, schémas, etc. Cette notion n'est pas très éloignée de celle de connaissances opérationnelles (Samurçay, \& Rogalski, 1992 ; Rogalski, 1995 ; Rogalski, \& Marquié, 2004), de « modèle opératif» ou de « structure conceptuelle de la situation » (Pastré, 2005 a et b). En effet, dans tous les cas, ce sont les connaissances organisées qui sous-tendent l'activité (les actions à réaliser). Il faut toutefois souligner que cette notion n'est pas l'objet central de son ouvrage.

7. Les outils d'analyse Procop (Poitrenaud, 1995) et Stone (Poitrenaud, 2001 ; Tijus, Cambon de Lavalette, Poitrenaud, \& Leproux, 2003) sont issus de cette lignée de recherches.

8. Dans une discussion de la notion de «schéma » - qui comprend des emprunts à Bartlett (pour les récits de situation), à la psychologie sociale (pour les stéréotypes) et aux schèmes de Piaget (comme structure assimilatrice de connaissance) - , Richard distingue avec Van Dijk les « schémas pragmatiques » qui relient des groupes de concepts, avec des relations du type tout-parties (que l'on pourrait considérer comme de même nature que celles qui relient les composantes d'un système, c'est nous qui commentons). Les relations entre perception et action (présentes dans les connaissances d'expérience) renvoient aux notions d'affordances de Gibson, aux schèmes piagétiens et aux images opératives d'Ochanine. 
utilisent la notion d'atome comme fil conducteur de leur texte pour expliciter les propriétés de ce type de concepts et leurs articulations à d'autres concepts au sein d'une théorie. Dans cette approche, orientée par les connaissances, les concepts sont des moyens de comprendre le monde et de définir des catégories empiriques. Les auteurs défendent l'idée que des progrès de la catégorisation dépendent des changements conceptuels, ce qui converge avec les positions de Vergnaud (1990), dont est pour partie issue la didactique professionnelle.

Dans l'approche de Thagard et Toombs (2005), la dimension représentative des concepts est centrale - dans un cours, Thagard" parle même de " schème représentationnel ». Comparativement, les concepts pragmatiques comprennent une dimension représentative et une dimension opérationnelle qui est centrale. Nous reviendrons sur cette question dans la discussion sur la place des concepts pragmatiques par rapport aux concepts techniques et scientifiques.

Toujours dans le cadre du courant de traitement de l'information, bien que ses travaux ne s'y résument pas, les apports de Leplat sur la formation portent au départ sur les habiletés (les «skills » chez Patrick, 1992), notamment dans les recherches initiales réalisées au CERP (Centre d'études et de recherches psychotechniques). On peut relever dans son ouvrage de 2002 une évolution vers une problématique s'intéressant aux compétences, dans laquelle la question des concepts est explicitement présente, même si les questions de conceptualisation n'ont pas été au cœur de ses recherches sur la formation. Enfin, Leplat, comme Richard (op. cit.), souligne les apports de Vergnaud et de Pastré dans la théorisation du développement des compétences.

L'ergonomie centrée sur l'activité (ou ergonomie francophone) ne s'est pas à proprement parlé intéressée au développement de l'adulte et au rôle de la conceptualisation dans le développement des compétences professionnelles. Toutefois, ses orientations, tant à propos de l'intervention, de l'analyse du travail que de la formation, produisent des résultats qui y participent. Nous en retiendrons trois points, à partir de la synthèse proposée par Falzon et Teiger (2004) :

- l'intervention ergonomique est un facteur de transformation des représentations des acteurs de l'entreprise (opérateurs, hiérarchie) à propos du travail ;

- l'analyse du travail est un outil pour les formés, qui a pour objectif de leur permettre d'acquérir une meilleure compréhension du travail et de ses effets, afin qu'ils puissent le transformer ;

- des dispositifs peuvent être conçus pour favoriser les activités réflexives et la construction de savoirs. L'objectif est alors d'assister les pratiques réflexives pour garder des traces des savoirs existants, qui ne sont d'ailleurs pas toujours explicites, et de favoriser l'élaboration de nouveaux savoirs.

Dans cette dernière orientation, les travaux s'appuient à la fois sur les apports d'Argyris et Schön (1978, 1996/2002), et sur ceux de Piaget (1974) à propos de la prise de conscience, les activités réflexives constituant un élément déterminant de l'expertise professionnelle. Reste que les thématiques centrales de l'ergonomie « de l'activité » ont concerné davantage les savoirs et ressources, produits de l'activité réflexive, que la conceptualisation en tant que telle et le développement des compétences professionnelles.

Le développement est en revanche d'emblée présent dans la ligne de recherches développée par Engeström (1987, 1994). S'appuyant sur les théories de l'activité, il met l'accent sur l'instruction et son rôle dans le développement, bien qu'il ne reprenne pas l'apport de Vygotsky (1934/1997) sur les concepts et leur développement. Et, si l'apprentissage, la représentation ou l'organisation des connaissances ne sont pas absents de ses travaux, la conceptualisation n'y est toutefois pas explicitement abordée.

On peut relever que c'est également le cas du courant de la cognition située, particulièrement représenté par Lave et Wenger $(1991)^{10}$. Ces auteurs définissent la conceptualisation comme un « événement

9. Les cours sont accessibles sur le site de l'auteur : http://cogsci.uwaterloo.ca/Biographies/pault.html (consulté en septembre 2006)

10. Nous n'avons mentionné que ces auteurs dans la mesure où ils constituent une référence à propos des apprentissages professionnels, dans le courant de l'action située. La position de la didactique professionnelle par rapport à ce courant 
particulier» rendant compte de «l'acquisition d'un principe abstrait» (p. 33). Que l'action soit située se traduit ici par le fait qu'une « représentation abstraite est sans signification tant qu'elle ne peut pas être spécifiée pour la situation en cours » (Ibid). Ces auteurs ne développent pas davantage le « principe abstrait » dont il s'agit, et n'explicitent pas de lien entre conceptualisation comme événement et représentation abstraite.

\subsection{2.- Dans les domaines de l'éducation et de la formation.}

Dans les domaines de l'éducation et de la formation, la notion de conceptualisation est une « entrée » récente, et essentiellement présente dans les développements théoriques engagés par Vergnaud et Pastré. Ainsi, un ouvrage dirigé par Ginsbourger, Merle et Vergnaud (1992) : « Formation et apprentissage des adultes peu qualifiés », qui présente le bilan de l'action de recherche éponyme débutée en 1987, est presque muet sur la conceptualisation : seul le chapitre de Pastré, « Apprentissage sur le tas et conceptualisation dans la conduite de machines automatisées » (1992b), y fait référence.

En France, les études et recherches sur la formation professionnelle et le développement chez l'adulte se sont organisées de manière dominante au sein des sciences de l'éducation. Elles y sont en général regroupées sous l'appellation « sciences de la formation ». Carré et Caspar ont coordonné un ouvrage récent (2004) qui propose un état des recherches dans le domaine. Les thèmes abordés dans l'ouvrage sont nombreux. Le développement de l'adulte y est traité dans une dimension très généraliste par Boutinet (2004) qui propose une réflexion sur la vie adulte à partir des histoires personnelles, des crises et transitions, de la temporalité et des défis de la vie adulte dans notre société. Dans ce même ouvrage, Pineau (2004) s'intéresse à l'apprentissage et au développement des adultes. Ces deux notions sont abordées à partir de celle d' " experiential learning ». L'auteur considère que l'expérience et les métaconnaissances jouent un rôle premier d'auto-régulation et d'auto-construction (p. 323). Dans son cadre, la conceptualisation, et la place des concepts dans l'apprentissage et le développement ne sont pas abordés. Toujours dans ce même ouvrage de référence, le développement cognitif de l'adulte est abordé par Vergnaud (2004), et plus succinctement par Pastré (2004b), à partir des questions de conceptualisation pour l'action.

Dans la littérature anglophone, une partie du courant « Educational research » s'intéresse au développement de l'adulte en reprenant un point de vue constructiviste dérivé des théories du développement cognitif de l'enfant. Ce courant intègre des apports des recherches réalisées à propos de l'acquisition des connaissances disciplinaires chez l'enfant et l'adolescent, ce qui conduit à mettre l'accent sur les changements conceptuels et la restructuration des connaissances plus que sur la place des processus de conceptualisation (on peut se référer à la synthèse de ces analyses du changement conceptuel dans Buty et Cornuéjols, 2002).

Dans ce cadre, dans une revue de question sur l'expertise professionnelle, Tynjälä et coll. (Tynjälä, Nuutinen, Eteläpleto, Kirjonen, \& Remes, 1997) soulignent la nécessité d'un rapprochement entre les travaux sur la formation initiale, qui concernent les apprentissages disciplinaires, et ceux qui ont été réalisés sur l'expertise professionnelle, afin de rendre compte et d'analyser l'apprentissage tout au long de la vie professionnelle. Ce faisant, ils précisent quelques enjeux pour ce courant de recherche, dont le fait de s'intéresser non plus à la nature de l'expertise mais à la façon dont cette dernière est acquise et de concevoir le développement des «connaissances expertes 》 du point de vue des changements conceptuels. Ainsi, le rôle de la conceptualisation dans le développement des compétences professionnelles y apparaît plutôt comme une orientation pour de futures recherches que comme l'objet de travaux existants.

Le courant récent « Work process knowledge » (WPK) (Boreham, Samurçay, \& Fischer, 2002 ; Fischer, Boreham, \& Nyhan, 2004) propose également une approche constructiviste prenant en compte conjointement ce qui est acquis au cours de la formation initiale et ce qui est acquis avec l'expérience professionnelle. L'historique de cette notion et de son utilisation est retracé par Boreham (2002) et Fischer et Boreham (2004). Ce courant propose en fait d'aborder la conceptualisation pour l'action

est détaillée dans Rogalski (2004). 
comme résultant d'un processus dialectique de résolution de contradictions entre ce que le sujet sait déjà, de manière « théorique », et les caractéristiques des situations auxquelles il doit faire face.

L'approche WPK présente une rupture par rapport au cadre théorique d'Anderson (1983, 1987 ; Anderson, \& Fincham, 1994 ; Harvey, \& Anderson, 1996) utilisé dans de nombreux travaux sur les apprentissages, cadre qui postule que l'expertise est le résultat d'une « compilation de connaissances ». La rupture est double : d'une part, les connaissances sur le processus de travail ne sont pas catégorisées en " déclaratives » ou " procédurales » et, d'autre part, deux types de connaissances s'articulent : par la réalisation de l'activité, les connaissances théoriques s'intègrent aux connaissances acquises par l'expérience. Les connaissances théoriques ne demeurent ainsi pas des connaissances « inertes ${ }^{11} »$, et les connaissances d'expérience ne sont plus simplement empiriques limitées aux situations courantes.

On peut toutefois noter qu'ici l'objectif est moins de s'intéresser aux processus de conceptualisation que de caractériser les différentes dimensions des connaissances sur le processus de travail, nécessaires aux opérateurs pour faire face aux changements contemporains dans les systèmes de travail (Boreham, Samurçay, \& Fischer, 2002), et de rendre compte des conditions nécessaires à leur acquisition en y incluant la formation initiale (Fischer, Boreham, \& Nyhan, 2004).

\section{2.-Développement des compétences et pragmatisation des connaissances théoriques}

Le développement des compétences implique, d'une part, l'acquisition des concepts pragmatiques du domaine et l'extension du réseau de relations entre concepts, indicateurs, et modes d'action, et d'autre part, une transformation du rapport aux connaissances théoriques enseignées en formation. Certes, la structure conceptuelle d'une situation est essentiellement organisée autour des concepts pragmatiques du domaine. Mais dans de nombreux domaines professionnels, la réalisation de l'activité de travail nécessite d'acquérir au préalable des connaissances théoriques, même si elles sont insuffisantes pour l'activité professionnelle. C'est par exemple le cas de la conduite de centrale nucléaire ou de la médecine.

En examinant la formation à la conduite de centrale nucléaire, Pastré (1999b, 2005a) propose d'appréhender ce qui est acquis au cours de la formation théorique qu'ont reçue les conducteurs débutants comme la constitution d'un « modèle cognitif». Ce modèle relève du registre épistémique et répond aux questions : « comment fonctionne l'installation? selon quelles relations de détermination entre les principales variables fonctionnelles ?» (Pastré, 2005a, pp. 74-75).

Les opérateurs observés sont des ingénieurs qui ont reçu, au cours de leur première phase de formation à la conduite de centrale, une formation théorique et technique de 13 semaines. La seconde phase de leur formation consiste à apprendre la conduite de centrale sur un simulateur pleine échelle, en bénéficiant du guidage des formateurs. L'auteur (1999b) relève que s'ils connaissent bien les principes de fonctionnement du système, ils ont du mal à faire face, en temps réel, à sa dynamique. Trois types de difficultés sont observées : ils prennent peu d'information sur l'ensemble du système et se contentent de celles qui relèvent de leur poste alors que les variables sont interconnectées; ils ont du mal à faire la différence entre des phénomènes de surface et des phénomènes représentatifs de la dynamique d'ensemble ; enfin, ils ne font pas la différence entre des « variables fonctionnelles (les buts de l'action) et des variables servant d'indicateurs » (p. 23).

Pastré en conclut que ce qu'ils apprennent au cours des simulations « sont les relations de signification reliant indicateurs, paramètres d'action et variables fonctionnelles » (1999b, p. 24), éléments qui constitueront un «modèle opératif ». Ce modèle relève du registre pragmatique et répond à la question « comment conduire la centrale ? ». Autrement dit, il permet de faire un diagnostic de la

11 «Les connaissances inertes peuvent être décrites comme une sorte de connaissances académiques » — « acquises dans des institutions comme l'école ou l'université », précisent les auteurs — « qui ne sont pas immédiatement utiles pour travailler et qui peuvent être difficiles à adapter aux différentes situations de travail » (Fischer, \& Boreham, 2004, p. 25, notre traduction). 
situation en cours pour agir. Il est composé d'indicateurs, de concepts organisateurs de l'action et de classes de situations (Pastré, 2005a).

Nous nous arrêterons sur la relation entre concepts organisateurs et indicateurs. Des informations disponibles (variables observées, mesurées, calculées) et certains indices vont être constitués en indicateurs par les opérateurs, qui les situent alors dans une relation de signification aux concepts organisateurs : « les indicateurs font sens par rapport aux concepts, qu'ils permettent d'évaluer » (op. cit., p. 77). C'est cette double relation, en lien avec les classes de situations constituées par les opérateurs, qui permet d'élaborer un diagnostic de la situation pour agir. D'après Pastré, ce qui est élaboré est « une sémantique de l'action ». Les connaissances théoriques sont l'objet d'une «pragmatisation »; elles deviennent des outils pour la conduite de l'installation, et cessent d'être dans une relation de connaissances « applicables » à la compréhension du fonctionnement de celle-ci ${ }^{12}$.

La constitution de nouveaux rapports entre «connaissances théoriques » et «connaissances pour l'action » a été l'objet de nombreuses recherches dans le domaine de la formation des médecins, en particulier autour, respectivement, de Patel (Patel, Evans, \& Groen, 1989 ; Patel, \& Kaufman, 1995 ; Patel, Kaufman \& Anocha, 2002) et de Boshuizen et Schmidt (Schmidt, Boshuizen, \& Hobus, 1988 ; Schmidt, \& Boshuizen, 1993 ; Boshuizen, Schmidt, Custer, \& van de Wiel, 1995 ; van de Wiel, Boshuizen, \& Schmidt, 2000).

Deux types de connaissances nécessaires à l'exercice de la médecine sont différenciées : les connaissances biomédicales, qui incluent des connaissances en anatomie, physiologie, biochimie, pathologie et pharmacologie, et les connaissances cliniques qui ont trait à la façon dont une maladie se manifeste chez les patients.

D'après Patel, les connaissances biomédicales et les connaissances cliniques constituent deux bases de connaissances distinctes. Elles ont leur propre structure, causale pour les connaissances biomédicales et associative pour les connaissances cliniques, et des modes de raisonnement propres leur sont attachés. Patel et al. (1989) suggèrent qu'avec l'acquisition de l'expérience professionnelle des relations sont établies entre ces deux bases de connaissances, mais ne précisent pas vraiment la nature de ces relations. En fait, Patel réserve le terme de connaissances conceptuelles aux seules connaissances scientifiques - qui permettent une « compréhension fonctionnelle»-, et parle pour les autres de « connaissances factuelles », et de " connaissances procédurales », liées au "savoir comment faire $\gg^{13}$ (Patel, Kaufman, \& Anocha, 2002). Son modèle des deux mondes de connaissances dissocie ainsi conceptualisation et action.

L'hypothèse défendue par Boshuizen et Schmidt est au contraire qu'il s'agit d'une seule base de connaissances : ils considèrent qu'avec l'acquisition de l'expertise les deux types de connaissances sont intégrés dans une seule base cohérente. L'utilisation répétée des connaissances pour le diagnostic et le traitement des patients en situation permet que « les réseaux élaborés de connaissances biomédicales acquises en formation initiale sont englobés dans des concepts cliniques de plus haut niveau », ce qui signifie que «les réseaux de connaissance des mécanismes physiopathologiques reliant les causes et les conséquences d'une maladies sont "capturés" par des concepts cliniques » (van de Wiel, Boshuizen, \& Schmidt, 2000, p. 328, notre traduction). Dans les termes de Schmidt et Boshuizen, il s'agit d'une « encapsulation » (Schmidt, Boshuizen, \& Hobus, 1988).

Dans des situations routinières, seuls les concepts « encapsulants » sont mobilisés, alors que dans des situations plus complexes l'ensemble du réseau est utilisé jusqu'aux connaissances les plus détaillées. En fait, les connaissances biomédicales sont mobilisées lorsque les connaissances cliniques

12. Richard (1983) avait introduit, à propos des systèmes informatisés, une distinction analogue en montrant l'existence d'une «logique de fonctionnement», utilisée par les concepteurs, distincte de la «logique d'utilisation ». Ce type de distinction apparaît en psychologie ergonomique dans les travaux de Bisseret et Enard (logique de construction et logique d'action, 1969-1970), mais aussi de Norman (1983) (modèle conceptuel, modèle mental). Hoc (1996) souligne l'existence de différents types de représentations « relatifs à la finalité des opérateurs » (p. 92 et sq.).

13. La différence que nous avons introduite plus haut entre deux faces de la compétence : savoir et pouvoir, ne correspond pas à la distinction introduite par Patel, classique dans une théorie de l'homme comme système de traitement de l'information, entre connaissances déclaratives et connaissances procédurales. Dans le cadre de la didactique professionnelle comme de l'approche WPK, ces connaissances relèvent toutes deux du savoir. 
s'avèrent insuffisantes ; pour les étudiants qui n'ont que peu ou pas d'expérience c'est souvent le cas, alors que pour des médecins plus expérimentés cela ne se produit que quand il s'agit de cas rares ou complexes.

En comparant différents sujets en cours de formation (des étudiants de première année en sciences jusqu'à des internes en médecine), Schmidt et al. (1988) montrent que l'encapsulation se produit par la réalisation de l'activité en situation — « as soon as the subjects are become exposed to actual patients »-, et qu'elle est plus importante chez les internes.

On trouve dans Boshuizen et al. (1995) un exemple simple d'encapsulation : la notion de « rétention d'urine »: elle est utilisée par les experts pour expliquer différents symptômes d'un patient. Elle « encapsule le processus de gonflement de la prostate qui obstrue le flux d'urine, l'urètre passant au travers de la prostate, conduisant à la rétention d'urine dans la vessie [...] et les changements anatomiques qui en résultent : distension de la vessie $[\ldots] »$ (notre traduction).

Schmidt et Boshuizen (comme Patel) travaillent essentiellement sur la structuration des connaissances sans aborder en elle-même la question de la conceptualisation. Cependant, bien que ce ne soit pas le cadre d'analyse des auteurs, la notion de « rétention d'urine » nous semble de la nature des concepts pragmatiques : schématisation opérative conduisant à constituer un certain nombre de symptômes en indicateurs et permettant le diagnostic, en lien avec les connaissances théoriques des pathologies. Ainsi, dans ces termes, l'encapsulation peut être interprétée comme un processus de constitution de concept pragmatique.

Dans un autre domaine, celui de la conduite des machines de navire marchand, un exemple d'encapsulation peut être identifié dans le document de formation des officiers, avec la notion de « chasse ». La chasse fait partie des opérations d'épuration de l'huile, pour la lubrification du moteur principal. « On appelle chasse, la séquence de nettoyage du bol de la centrifugeuse par ouverture et vidange du bol. Cette opération sert à évacuer les impuretés les plus lourdes, qui, par le phénomène de centrifugation, sont projetées à la périphérie du bol où elles s'accumulent. L'ouverture, en marche, du couvercle du bol, libère ces boues qui sont alors évacuées vers la caisse à boues » (Percier, 1990). La notion de chasse, qui relève de l'action, encapsule le «pourquoi » de l'action - le but de l'action étant lié à des connaissances techniques de mécanique des moteurs : la nécessité de lubrification -, le « comment» qui réfère au phénomène dynamique de centrifugation; la précision « en marche " pour l'ouverture du bol est implicitement justifiée par des connaissances générales de dynamique.

Les exemples de Pastré, et ceux de Boshuizen et Schmidt soulignent que la réalisation de l'activité permet de réorganiser des connaissances et d'élaborer de nouvelles relations de signification. On retrouve ici la relation dialectique entre les connaissances dont dispose l'opérateur, dont les connaissances «théoriques » acquises en formation initiale, et la confrontation aux caractéristiques des situations, relation soulignée ailleurs par Fischer et Boreham (2004). Dans la confrontation aux caractéristiques des situations, deux mouvements de transformation de la relation entre les connaissances théoriques et les concepts pragmatiques peuvent être différenciés : d'une part, les concepts théoriques vont acquérir un nouveau sens, ils sont " pragmatisés ${ }^{14}$ » en s'inscrivant dans une sémantique de l'action, les connaissances théoriques deviennent ainsi directement opératives et, d'autre part, des concepts pragmatiques vont être constitués.

L'exemple de l'apprentissage de conduite de centrale, comme celui de la médecine, mettent en évidence que, dans certains domaines, des connaissances théoriques « suffisantes » de l'opérateur sont un préalable pour pouvoir être mis en situation. Ce n'est toutefois pas la seule voie de constitution des concepts pragmatiques. Ils peuvent aussi s'acquérir par la réalisation de l'activité en situation, «sur le tas ", avec le concours de la communauté professionnelle : les travaux de Pastré sur la plasturgie en sont un exemple (1992a, 1994, 1997, 2004a). L'intervention de l'analyste amenant l'opérateur à réfléchir sur son action et mettant en évidence la structure conceptuelle de la situation, par exemple, peut constituer un facteur de développement. L'utilisation de la notion de « chasse » dans la conduite

14. Ce terme est emprunté à Pastré (2005a) pour rendre du compte du fait qu'un concept théorique acquis en formation et présenté en tant que tel est transformé par la réalisation de l'activité en situation. 
de machine pourrait être acquise de cette manière, toutefois le contrôle de la qualité de la " chasse » (par le moment d'ouverture du couvercle) relève de connaissances de mécanique.

Pour avancer dans la discussion des processus postulés dans la transformation des relations entre types de connaissances, de la place relative de ces connaissances et du processus de conceptualisation lui-même, nous allons maintenant discuter plus directement des concepts : concepts scientifiques et/ou techniques (que nous regrouperons souvent sous le vocable de concepts théoriques), concepts quotidiens, et concepts pragmatiques.

\section{3.- Rapports et différences entre types de concepts (scientifiques / techniques - quotidiens - pragmatiques - en-acte)}

Dans un premier temps, nous nous attacherons à différencier concepts scientifiques, techniques et pragmatiques, puis nous présenterons des exemples de leurs relations dans des domaines professionnels. Nous aborderons ensuite les relations entre concepts théoriques (scientifiques et techniques), concepts quotidiens et concepts pragmatiques. Enfin, nous discuterons des relations entre concepts pragmatiques et théorèmes et concepts en-acte issus de la théorie des champs conceptuels de Vergnaud (1990).

\section{1.- Relations entre les concepts pragmatiques et les concepts théoriques}

Pour aborder les relations entre les concepts pragmatiques et les concepts théoriques (scientifiques et techniques) nous nous intéresserons d'abord aux relations entre concepts scientifiques et techniques (leurs similarités, relations et différences), nous discuterons ensuite des différences entre concepts pragmatiques et techniques, et nous présenterons des exemples de relations entre concepts théoriques et concepts pragmatiques.

\subsection{1.- Concepts scientifiques et concepts techniques}

Science et technique produisent des connaissances - pour la technique Vincenti (2001) utilise le terme de «ingeneering science»—, et prennent la réalité comme objet: il s'agit d'identifier, de caractériser, de modéliser « des propriétés relativement stables et manifestant des comportements prévisibles » (Vérillon, 2002). Dans les deux cas, la validité est un critère pour juger des savoirs produits (Gibbons, 2000 ; Rogalski, \& Veillard, 2002) et leur légitimité est débattue au sein du domaine (Rogalski, \& Samurçay, 1994). Ainsi, les concepts techniques peuvent être regardés comme « issus d'une démarche systématique de construction sociale, parente de la démarche scientifique » (Samurçay, \& Rabardel, 2004, p. 178).

Les connaissances scientifiques et techniques interagissent : une connaissance technique peut constituer un «challenge» pour la science qui a à l'expliquer, et inversement les évolutions des cadres théoriques produites dans le domaine scientifique peuvent constituer un "challenge " pour le domaine technique (Gibbons, 2000). Ainsi, pour un ingénieur, l'utilisation d'une théorie dans un cadre expérimental peut avoir pour objectif de vérifier qu'elle fonctionne bien comme un outil utile pour la conception (Vincenti, 2001).

Aussi, la plupart des philosophes des technologies s'accordent pour établir une différence entre les concepts scientifiques et techniques (de Vries, 2003). Constant (1980) et Vincenti (1990) ont souligné que si la connaissance scientifique est utilisée par les ingénieurs dans leurs activités de conception, le corps de connaissances qu'ils mobilisent diffère de la connaissance scientifique (de Vries, op. cit.). Ainsi, Vincenti (op. cit.) a catégorisé les différents types de connaissances utilisés par les ingénieurs de l'aéronautique. Elles sont synthétisées par de Vries (op. cit., p. 3) : « des concepts fondamentaux (principes opérationnels et configurations "normales"), des spécifications et des critères de design, des outils théoriques (mathématiques, raisonnements, lois de la nature), des données quantitatives 
(descriptives et prescriptives), des considérations pratiques et des instruments de design ».

Les visées dominantes de la science et de la technique diffèrent. Vérillon (2002) rappelle que si science et technique partagent un même rapport à la réalité (laquelle est prise comme objet), ce n'est pas avec la même visée : pour la science le monde est « objet à connaître » alors que pour la technique, il est « objet à utiliser». Dans le domaine scientifique, l'objectif est en effet de produire de l'information et de la connaissance. L'action est au service de cette production (Sigaut, 1991). Comparativement, du côté de la technique, le réel, objet à utiliser, est analysé pour " produire un effet intéressant, un état avantageux ou un artefact utile » (Vérillon, op. cit.). La connaissance du réel est ici au service de l'action (Sigaut, 1991), de la pratique, telle que la définit Vicenti (2001). De ce point de vue, schématiquement, deux visées peuvent être différenciées : visée épistémique pour la science, visée pragmatique pour la technique.

\subsection{2.- Concepts techniques et concepts pragmatiques}

Les concepts pragmatiques sont essentiellement liés à l'efficacité de l'action, ainsi comme les concepts techniques, leur visée est à dominante pragmatique. Sont-ils pour autant une catégorie de concepts techniques?

Une première différence entre concepts pragmatiques et concepts techniques concerne le domaine de validité et le rapport validité-efficacité-pertinence. En effet, d'une part, les concepts pragmatiques sont au cœur des savoirs professionnels et permettent d'agir efficacement dans des classes de situations d'un domaine professionnel donné. Leur degré de validité est donc moins étendu que celui des concepts techniques. D'autre part, «à la validité des savoirs scientifiques et techniques répond la pertinence et l'efficacité des savoirs professionnels » (Rogalski, \& Veillard, 2002, p. 94).

On peut ici rajouter que dans un domaine professionnel, on n'entreprend pas de démarche systématique de validation des concepts pragmatiques, contrairement aux domaines scientifiques et techniques. Par exemple, les opérationnels de la lutte contre les feux ne cherchent pas à justifier les concepts pragmatiques d'axe de propagation, ou de vitesse de propagation du feu, ils s'en servent pour anticiper et lutter contre le feu.

Ce n'est pas sans lien avec le fait que les concepts pragmatiques permettent de penser et de comprendre pour agir en situation, ici et maintenant. Alors que comparativement, les concepts techniques sont plutôt issus d'une conduite de détour, de la volonté explicite de théoriser suffisamment un phénomène pour s'assurer de la cohérence d'ensemble, de l'amplitude du domaine de validité et de l'utilité du concept technique.

Enfin, les concepts pragmatiques sont inséparables de l'organisation de l'action. C'est ce dont rend compte la notion de «structure conceptuelle de la situation ».

Pour rendre compte de ces différences, nous reprendrons un exemple du domaine de la lutte contre les feux de forêt :

- Dans le domaine technique, les recherches sur le feu posent la question, non résolue, de savoir comment la vitesse du feu dépend de la vitesse du vent.

- Pour les opérationnels de la lutte contre les feux de forêt, de toute façon la conjonction des effets de vitesse du feu et de vitesse du vent doit être prise en compte. Peu importe les relations exactes entre la vitesse du vent et la vitesse du feu ${ }^{15}$. Ils savent très bien que la vitesse du feu croît avec la vitesse du vent (les autres variables étant égales).

Une dernière différence entre concepts pragmatiques et concepts techniques concerne la formation. Quand il s'agit d'apprentissages disciplinaires (scientifiques ou techniques) ce qui doit être enseigné est déjà connu - et socialement reconnu —, et doit être transposé pour être enseigné (Chevallard, 1985/1991), alors que cela doit être identifié par l'analyse de la situation de travail

15. En considérant la vitesse du feu $\left[\mathrm{V}_{(\mathrm{f})}\right]$ et la vitesse du vent $\left[\mathrm{V}_{(\mathrm{v})}\right]$, l'une des questions est de savoir si « $\mathrm{V}_{(\mathrm{f})}=\mathrm{K} \sqrt{ } \mathrm{V}_{(\mathrm{v})}$ » ou si « $\mathrm{V}_{(\mathrm{f})}=\mathrm{K} \mathrm{V}_{(\mathrm{v})}{ }^{2} »$, selon un modèle de 0,5 à 2. 
quand il s'agit d'un domaine professionnel particulier. Il s'agit alors d'identifier la structure conceptuelle de la situation de travail (Pastré, 1999b), ou plus largement les « savoirs professionnels de référence » (Rogalski, \& Samurçay, 1994), qui ne se réduisent pas à des concepts scientifiques et/ou techniques déjà déterminés ${ }^{16}$.

\subsection{3.- Des exemples de relations entre les concepts théoriques et les concepts pragmatiques}

Dans l'activité professionnelle, les relations entre les concepts théoriques et pragmatiques ne sont pas univoques. Trois aspects de leurs relations sont présentés ici : des concepts pragmatiques existent dans des domaines pour lesquels les conceptualisations théoriques sont insuffisantes; ils peuvent également s'inscrire dans des relations avec des concepts théoriques; et enfin, dans certains cas, un même terme peut renvoyer à un concept scientifique, technique ou pragmatique. En effet, ils partagent des significations mais ils ne sont pas situés dans les mêmes cadres, ni leurs usages orientés par la même finalité.

\section{Insuffisance des modélisations théoriques}

Dans un certain nombre de domaines professionnels les modélisations théoriques existantes peuvent s'avérer insuffisantes pour rendre compte et expliquer les processus et phénomènes en jeu. Nous prendrons pour exemple le domaine de la lutte contre les feux de forêt.

La lutte contre les feux de forêt est un cas de gestion opérationnelle, une des missions-clés des officiers sapeurs-pompiers de la sécurité civile dans le sud-est de la France. Il s'agit d'élaborer et de conduire les opérations rendues nécessaires par des sinistres, qui sont des environnements dynamiques, à risque, potentiellement de grande ampleur. Élaborer des modèles des évolutions et des modèles de l'effet des actions sur ces sinistres est une nécessité, comme Bainbridge (1988) l'avait souligné à propos du contrôle de processus. Le caractère « ouvert » de ces environnements dynamiques tient à la modélisation limitée qui peut en être faite en termes de concepts scientifiques ou techniques.

En effet, d'une part, le feu en tant que tel est un phénomène qui reste extrêmement complexe. Les modèles calculables existant portent uniquement sur des conditions homogènes quant au combustible et au comburant, et sur des dimensions spatio-temporelles limitées. Bien sûr en situation réelle, ces conditions d'homogénéité n'existent pas. D'autre part, les modèles de simulation élaborés, qui s'appuient sur le développement « local » d'un feu et qui ont été évalués par rapport à des feux réels, prennent en compte un nombre considérable de paramètres, ce qui en limite la possibilité d'emploi en situation d'action. Ces paramètres concernent la topographie, la végétation (nature, humidité du sol et des plantes, distribution topographique), et les éléments météorologiques (vent, température, hygrométrie). Certains peuvent varier à la fois dans l'espace et le temps court, ce qui les rend difficiles à évaluer.

Ce type de modélisation peut permettre des études de prévention et de formation, mais en situation d'action, l'identification de l'état et de l'évolution possible s'appuie sur un modèle opératif, articulant un ensemble de concepts pragmatiques.

En fait, le modèle qui est utilisé en situation est basé sur une représentation schématique des propriétés des feux problématiques, centrées sur une image opérative du feu (Ochanine, 1978). Ce modèle s'appuie sur un ensemble organisé de concepts pragmatiques largement partagés par la communauté, et par ailleurs accessibles à des non-professionnels. Nous en donnons ici quelques nouveaux exemples : «tête » ou « front du feu », «flancs », « arrière » ou « cul du feu » dérivent clairement d'une métaphore du feu comme animal menaçant une « zone du feu » et des « points sensibles ».

Un élément important du modèle est l'invariance de la forme du feu, qui garde la même image

16. Bien sûr, dans de nombreux domaines professionnels, ce qui doit être enseigné est connu. La mise en évidence des savoirs professionnels de référence reste alors pertinente dans un objectif d'amélioration des situations de formation. Par ailleurs, nous considérons que la structure conceptuelle des situations fait partie des savoirs professionnels de référence, qui comportent également par exemple des méthodes de traitement des problèmes. 
opérative, cependant que change sa taille absolue. Ces concepts pragmatiques sur l'objet de l'action permettent de définir un référentiel constant indépendamment des positions qu'occupent les acteurs opérationnels ${ }^{17}$, et indépendamment des formats de désignation des lieux. Ils permettent d'identifier aussi bien les points sensibles - menacés par la propagation dans la «zone du feu »-, que les potentialités d'action liées aux propriétés de la zone d'intervention — zone du feu et zone de déplacement des moyens.

\section{Existence de relations entre concepts théoriques et concepts pragmatiques}

Comme nous l'avons noté à partir des exemples de formation à la conduite de centrale nucléaire ou de formation à l'exercice de la médecine, les connaissances théoriques peuvent constituer un préalable à la réalisation de certaines activités de travail, cependant qu'avec l'acquisition de l'expérience, elles seront transformées (encapsulation, pragmatisation).

Dans une revue de questions sur les articulations entre les différents types de connaissances, Rogalski et Veillard (2002) citent de nombreux travaux sur l'utilisation de savoirs disciplinaires dans les activités professionnelles. Si dans l'ensemble ce type de recherches constate un décalage entre savoirs disciplinaires et professionnels qui pourrait être mis en relations avec l'orientation épistémique versus pragmatique de l'activité, certains travaux mettent aussi en évidence des conceptions erronées qui sont un obstacle à la réalisation d'une activité professionnelle efficace.

Par exemple, Hahn (1999) s'est intéressée à la façon dont des élèves en formation initiale de vendeur utilisent des outils mathématiques de proportionnalité pour calculer des prix « toutes taxes comprises » (TTC) ou « hors taxe» (HT). Elle relève des conceptions erronées qui se traduisent par des procédures de type " pourcentage additif ». Par exemple, à partir du prix TTC (100€) et de la taxe $(5,5 \%)$, on doit calculer le prix HT. L'erreur consiste à soustraire 5,5 à 100. On obtient 94,50€ (au lieu de $100 € X$ 100/105,5 = 94,80 €). La différence n'est pas sensible dans l'échange commercial mais posera quelques problèmes à la comptabilité.

Les besoins de connaissances théoriques peuvent se manifester dans des situations critiques (Rogalski \& Veillard, op. cit.). Une recherche sur les risques électriques dans la livraison de béton prêt à l'emploi sur des chantiers nous permet de l'illustrer. L'analyse cognitive de la tâche, réalisée en utilisant des analyses d'accidents, des entretiens avec les opérateurs et l'examen de la réglementation du domaine, conduit à différencier deux types de situations incidentelles (Vidal-Gomel, Olry, Jeanmougin, \& Lanoë, 2005) :

Si l'engin de livraison (muni d'un tapis de livraison ou d'un bras articulé) entre en contact avec une ligne électrique aérienne haute ou moyenne tension et si l'opérateur se trouve à l'intérieur de son camion, il ne doit pas descendre de sa cabine. Il doit attendre la fin des réenclenchements automatiques de la ligne électrique pour descendre ${ }^{18}$. Dans le cas contraire, il peut toucher la masse métallique sous tension en étant en contact avec le sol. Le risque est particulièrement important au moment où l'opérateur descend la dernière marche du marchepied, et sauter n'est pas une solution, il peut se trouver dans une autre catégorie de situation (que nous présentons ci-après) sans l'avoir identifiée. Soumis à une différence de potentiel, il est alors électrisé ou électrocuté19.

- Si le contact conduit à la création d'un courant de défaut (la ligne électrique a été arrachée et touche le sol, par exemple) et si l'opérateur se trouve à l'extérieur de sa cabine, il doit prendre

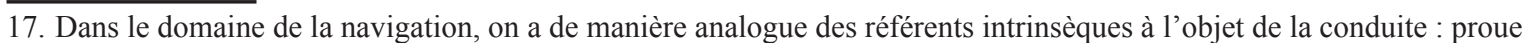
et poupe (avant / arrière), et surtout tribord et babord, qui évitent les compositions des références « droite / gauche » par rapport aux repères variables des membres d'équipage.

18. Comme pour tout réseau électrique, en cas d'incident d'origine électrique, une protection se déclenche et met le réseau hors tension. Pour les lignes électriques aériennes, plusieurs remises sous tension sont effectuées automatiquement avant la coupure définitive. Cela permet de ne pas mettre hors tension une portion importante du réseau à chaque incident électrique mineur (chute d'une branche d'un arbre sur une ligne, par exemple).

19. Les conséquences sont toujours graves étant donné les caractéristiques du danger : électricité haute ou moyenne tension. 
en compte la «tension de pas ». En effet, du fait de la résistivité électrique du sol, le courant « diffuse » autour du point d'impact et génère un « gradient de potentiel ». Ainsi un risque de choc électrique par tension de pas peut exister « entre deux cercles de gradient de potentiel distants de 0,80 $\mathrm{m} »$ (Dugrillon, 2002), ce qui représente une distance de l'ordre de celle qui est normalement franchie d'un pas, par un adulte. Dans une telle situation, il faut s'éloigner du point d'impact en faisant des petits pas ou des petits bonds.

Les procédures de récupération pertinentes dans ces deux types de situations ne peuvent pas s'acquérir en situation, et leur transmission par des pairs peut s'avérer problématique. En effet, leur mise en œuvre ne relève pas uniquement de l' " application » et nécessite la compréhension de la «théorie du risque » (Mayen, \& Savoyant, 1999) du domaine professionnel concerné. Cette théorie comprend plusieurs notions en relation (par exemple la notion de tension de pas fait intervenir la notion de différence de potentiel), dont toutes, à l'exception de la tension de pas, appartiennent au domaine de l'électricité, champ de connaissances scientifiques de physique.

\section{Une même notion peut fonctionner comme concept scientifique, technique ou pragmatique.}

Nous prendrons un dernier exemple pour illustrer les relations multiples entre concepts pragmatiques, techniques et scientifiques, celui de la parallaxe, qui permet d'illustrer ces relations selon un axe d'extension du degré de validité du concept : du concept pragmatique au concept scientifique ; axe d'extension décrit par Samurçay et Rabardel (2004). Cet exemple rend également compte de l'utilisation d'une notion en fonction de la finalité de l'activité du sujet (selon un continuum allant d'une visée épistémique à une visée pragmatique).

— La parallaxe comme concept scientifique.

La parallaxe est un déplacement angulaire apparent, par rapport à un objet de référence, d'un corps observé à partir de deux points différents (Villemin, 2005). C'est un effet de perspective sensible lorsqu'on observe un même objet depuis deux points éloignés. Le phénomène dont la parallaxe rend compte s'observe facilement : en regardant un poster, par exemple, tout en mettant l'index dans l'axe de vision, en fermant alternativement chacun des yeux, on constate que l'index change de place par rapport aux dessins du poster (Ibid.).

La parallaxe est un concept mathématique qui est très utilisé en astronomie : son calcul donne une valeur de la distance des objets célestes. Elle est liée au mouvement de la Terre autour du Soleil, qui conduit à une variation de la direction sous laquelle on voit un objet. Proxima du Centaure, qui est l'étoile la plus proche de la terre (43000 milliards de $\mathrm{km}$ ) en dehors du Soleil, a une parallaxe de 0,772 seconde d'arc, soit la plus grande possible pour une étoile (Ibid.).

Le calcul de la parallaxe permet donc d'acquérir des connaissances sur les objets du système solaire. L'objectif est épistémique. Il implique, notamment, d'utiliser le théorème de Thalès qui s'appuie sur des propriétés des droites parallèles. En astronomie, outre les connaissances propres au domaine, le calcul de la parallaxe inclut également des connaissances en trigonométrie (pour plus de détails voir le site « Tout pour la science »).

- La parallaxe comme concept technique

Nous nous intéressons ici au domaine de la photographie. Les connaissances nécessaires à la prise en compte et à l'explication des questions de parallaxe relèvent de l'optique géométrique et des propriétés des appareils de photographie. En particulier, la correction de la parallaxe en photographie est théorisée (voir encadré 1 en annexes). Il ne s'agit donc plus de connaître la parallaxe, son calcul ou son rapport à la connaissance de la position des objets lointains, comme nous l'avons noté précédemment.

— La parallaxe comme concept pragmatique

Restons dans le domaine de la photographie. Les erreurs de parallaxe sont connues et définies: « c'est la différence entre la portion d'espace vue dans le viseur et l'angle de champ de l'objectif» 
(d'après le site du « Guide de la photographie panoramique »).

Aussi pour faire des photographies panoramiques ou des paysages, il faut « veiller au cadrage quand on effectue une mise au point rapprochée. Il y a une différence de cadrage entre ce que l'on voit dans le viseur - souvent au-dessus de l'appareil - et ce qui est vraiment photographié à cause de la parallaxe. Plus le viseur est éloigné du film et plus cet effet sera prononcé. Cet inconvénient n'est vraiment pas grave dans le cas de certains modèles car on peut enlever le viseur de son support pour le placer à la hauteur du film [...]. Ce point est également à prendre en compte si l'on réalise des photos panoramiques par assemblage, à la prise de vue mais aussi pendant la recherche de la pupille d'entrée (souvent appelée point nodal)» (op. cit.).

La notion de parallaxe est uniquement utilisée ici pour rendre compte de la différence entre ce que l'on voit dans le viseur et ce qui sera photographié. Elle n'implique pas de calcul, on ne cherche pas à « connaître » la parallaxe, ni à expliquer l'ensemble des phénomènes en jeu pouvant rendre compte des problèmes de parallaxe dans le domaine de la photographie, comme précédemment. En revanche, il est nécessaire au photographe de se représenter cette distance, de la prendre en compte dans la composition de la photographie et, éventuellement, de mettre en ouvre des actions correctrices - par exemple, avec certains appareils, on peut enlever le viseur de son support. C'est bien ici l'efficacité de l'action qui est en jeu.

Par ailleurs, il ne s'agit pas d'un concept-en-acte ou d'un théorème-en-acte. En effet, les problèmes liés à la parallaxe sont bien connus dans le milieu de la photographie et les photographes sont en mesure de les expliciter (un exemple est présenté dans l'encadré 2, en annexes). De notre point de vue, dans ce cas, la parallaxe est un concept pragmatique.

\section{2.- Relations avec les concepts quotidiens}

La notion de concept quotidien (ou spontané) est développée par Vygotski dans « Pensée et langage » (1934/1997). Il consacre une part importante de l'ouvrage aux relations entre concepts scientifiques et concepts quotidiens, et à leurs rôles dans le développement des enfants.

Les concepts quotidiens ont été l'objet de nombreux travaux de psychologie de l'éducation et de didactique disciplinaire, qui les ont considérés sous des thématiques variées, comme « représentations naïves » (Tiberghien, 2002), « misconceptions » (Chi, 2005) ou « obstacles à l'apprentissage » (Closset, 1989). Notre propos n'est pas d'en rendre compte ou de proposer une synthèse de ce type de travaux, mais nous nous y réfèrerons afin de préciser les caractéristiques des concepts quotidiens au-delà des apports de Vygotski, et les différences entre concepts scientifiques ${ }^{20}$, quotidiens et pragmatiques.

\subsection{1.- Caractéristiques des concepts quotidiens et différences avec les concepts scientifiques}

D'après Vygotski (1934/1997), les concepts quotidiens se forment spontanément au cours de l'expérience personnelle via l'interaction sociale, sans pour autant que cette interaction ait comme objectif l'acquisition d'un ensemble organisé de concepts. Il les différencie des concepts qui sont acquis dans le cadre scolaire et qu'il considère comme des concepts " scientifiques », ce qui n'est pas sans ambiguïté puisque les concepts scientifiques sont alors assimilés à l'ensemble des notions enseignées en classe. Nous retiendrons de cette différenciation que les concepts quotidiens sont acquis sans qu'il y ait eu intention didactique d'un tiers, contrairement aux concepts enseignés, dont font partie les concepts scientifiques (Rogalski, 2006).

Pour Vygotski, concepts quotidiens et concepts scientifiques sont dans une relation dialectique. Les concepts quotidiens sont nécessaires pour acquérir des concepts scientifiques, ils permettent de leur donner un premier sens, issu de l'expérience. En même temps, l'acquisition des concepts scientifiques transforme les concepts quotidiens. Cette relation dialectique intègre, par exemple, le fait que

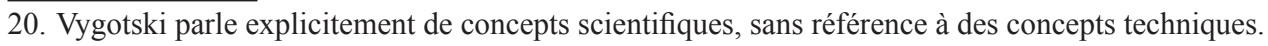


l'identification de contradictions dans l'utilisation de concepts quotidiens peut contribuer à donner du sens à un concept scientifique, qui en quelque sorte lève la contradiction.

Cette relation dialectique ne suffit toutefois pas à rendre compte des interactions complexes entre concepts scientifiques et quotidiens. Ainsi, des conceptions naïves peuvent cohabiter, chez un même sujet, avec des conceptions plus élaborées acquises dans un cadre scolaire, sans qu'il semble y avoir interférences entre les deux ; " chaque conception n'étant mobilisée que dans une situation précise » (Joshua, \& Dupin, 1993, p. 159). De plus, certains concepts scientifiques n'ont pas de précurseur dans les concepts quotidiens. C'est, par exemple, le cas du concept d'inertie (Viennot, 1996). Présentant des travaux sur ce thème, Viennot (op. cit) identifie même une « obstruction épistémologique » à l'appropriation de ce concept ; obstruction liée à une représentation du type «les objets gardent la mémoire de leur passé ». D'autres obstacles tiennent aux caractéristiques de ce qu'est une « entité théorique »- elle est abstraite et non observable, comme le soulignent Thagard et Toombs (2005) —, ou au fait que les sujets introduisent du réalisme, des relations temporelles et/ou événementielles quand il s'agit de relations causales (Viennot, op. cit.).

Un exemple classique d'étude montrant ce que Viennot appelle « l'introduction du réalisme » part de la situation suivante : on dit au sujet que l'on projette l'image d'une bougie sur un écran au travers d'une lentille et que la lentille comporte un trou. Le modèle spontané, très répandu chez les sujets, est que la lentille « agit » pour « donner » l'image. Ainsi puisqu'il y a un trou dans la lentille, il y a un trou dans l'image projetée.

Pour analyser les concepts quotidiens Vygotski (op. cit.) les oppose aux concepts scientifiques. Ainsi il précise plusieurs caractéristiques des concepts quotidiens : ils ont une portée locale, ils sont isolés et sont non conscients. Si l'on peut considérer que les concepts quotidiens ont une portée locale au regard des concepts scientifiques, il est nécessaire de discuter de leur caractère isolé et de leur caractère non conscient (Rogalski, 2006) : les concepts quotidiens ont-ils systématiquement ces caractéristiques ? En effet, d'une part, les recherches sur les conceptions communes ont montré qu'il existe des zones de cohérence dans ce savoir et qu'il ne s'agit pas d'un « savoir en miette » (Viennot, 1996), comme pourrait le laisser supposer le caractère isolé que Vygotski attribue aux concepts quotidiens. D'autre part, les concepts quotidiens peuvent être explicites, mais aussi fonctionner « en acte », sans que le sujet en ait conscience, sans qu'ils soient verbalisables. Dans ce cas, nous considérons qu'il s'agit de « concepts-en-acte » au sens de Vergnaud (1990).

\subsection{2.- Concepts quotidiens et concepts pragmatiques}

Plusieurs caractéristiques nous permettent de différencier concepts quotidiens et concepts pragmatiques.

Nous avons discuté du caractère isolé des concepts quotidiens en retenant qu'ils pouvaient être isolés mais que ce n'était pas systématique. Comparativement, les concepts pragmatiques sont toujours inscrits dans un réseau de relations comprenant des variables et d'autres concepts et, avec l'expérience, ce réseau est plus étendu (Samurçay, \& Pastré, 1995). D'autre part, les concepts quotidiens se forment spontanément au cours de l'expérience, avec un faible concours du langage (Vygotski, op. cit.), contrairement aux concepts pragmatiques qui sont transmis par la communauté professionnelle, notamment sous des formes langagières. Enfin, les concepts quotidiens ne sont pas forcément orientés par un objectif d'action, contrairement aux concepts pragmatiques. Par exemple, les enfants ont des représentations « naïves » de phénomènes complexes bien qu'ils ne les aient pas vus, n'aient pas à agir dessus ou avec.

Une dernière caractéristique des concepts quotidiens nous semble constituer un parallèle avec les concepts pragmatiques : ils sont un moyen de donner du sens aux concepts scientifiques. Les exemples que nous avons précédemment présentés d'encapsulation ou de pragmatisation des concepts avec constitution d'une sémantique de l'action, dans les domaines de la formation à la médecine ou à la conduite de centrale nucléaire, en rendent compte. 


\section{3.- Concepts pragmatiques et concepts-en-acte dans une théorie de l'action}

La notion de concept pragmatique s'inscrit dans la lignée des apports de Vergnaud (1985, 1990). L'accent y est mis sur la conceptualisation et le rôle des concepts-en-acte et des théorèmes-en-acte, invariants opératoires et cœur de la représentation. Avant de discuter des différences entre concepts pragmatiques et concepts-en-acte, nous rappellerons d'abord les notions de théorèmes-en-acte et de concepts-en-acte.

\subsection{1.- Concepts-en-acte et théorèmes-en-acte, des invariants opératoires}

« La théorie des champs conceptuels » (Vergnaud, 1900) a été développée dans un contexte d'études sur les acquisitions des élèves en mathématiques. Les notions de concepts-en-acte et de théorèmesen-acte, qui y sont développées, permettent de rendre compte des connaissances des élèves en les rapportant au champ conceptuel considéré - Vergnaud les a d'ailleurs formalisés dans les termes mêmes du savoir dont l'acquisition est visée. Ce faisant, il a pu mettre en évidence que l'activité des élèves est orientée par des invariants opératoires et qu'elle ne pouvait donc pas être uniquement analysée en termes de connaissances justes et d'erreurs. L'expression «en-acte » souligne que ces invariants peuvent n'être exprimés que dans l'action.

Vergnaud (1990, p. 142-143) différencie plusieurs types d'invariants opératoires, dont des invariants de type « propositionnel » et de type « fonction propositionnelle ».

— Des invariants de type propositionnel :

Les théorèmes-en-acte appartiennent à cette catégorie. « Proposition tenue pour vraie sur le réel » par le sujet, un théorème-en-acte peut être vrai ou faux.

Vergnaud en donne l'exemple suivant : « Entre 5 et 7 ans, les enfants découvrent qu'il n'est pas nécessaire de tout recompter pour trouver le cardinal d'une collection $\mathrm{A} \cup \mathrm{B}$, si on a déjà compté $\mathrm{A}$ et $\mathrm{B} »$. On peut exprimer cette connaissance par un théorème-en-acte :

« $\operatorname{Card}(\mathrm{A} \cup \mathrm{B})=$ Card $(\mathrm{A})+$ Card $(\mathrm{B})$ pourvu que $\mathrm{A} \cap \mathrm{B}=\varnothing$ » (Ibid.).

- Des invariants de type fonction propositionnelle :

Ils constituent « les briques indispensables à la construction de propositions ». Ce sont des conceptsen-acte. Si nous reprenons l'exemple précédent, il s'agit des concepts de collection, de cardinal, de réunion de collections et d'addition numérique. On pourrait ici rajouter que les enfants savent assez tôt que Card(A) + Card (B) = Card (B) + Card (A). La commutativité de l'addition est d'abord utilisée comme un théorème-en-acte; elle ne deviendra explicite qu'avec l'enseignement, au niveau du secondaire, et apparaîtra plus tard seulement comme un concept algébrique général (une propriété des opérations binaires).

Les exemples précédents permettent d'identifier quelques éléments du champ conceptuel des structures additives : il est composé des notions de collection, de nombre, d'addition, de réunion des collections, etc., et de leurs relations.

Définir un invariant opératoire comme une proposition que le sujet tient pour vraie sur le réel introduit une ambiguïté, que l'on peut résumer par la question suivante : toutes nos connaissances sont-elles des invariants opératoires ? Dans un article de 1985, Vergnaud précise que les invariants opératoires sont « des objets, propriétés et/ou relations » qui nous permettent de « découper le réel » pour « organiser l'action» (p. 145). Ils « constituent le noyau dur de la représentation, celui sans lequel ni les inférences, ni les prédictions, ni les signifiants n'ont de sens » (p. 250). Cela ne préjuge pas de différences éventuelles entre types d'invariants opératoires - il peut s'agir de concepts scientifiques, techniques, pragmatiques, ou quotidiens. 


\subsection{2.- Concepts pragmatiques et concepts-en-acte : quelles différences ?}

La filiation entre les travaux de Vergnaud $(1985,1990)$, qui mettent l'accent sur les théorèmesen-acte et les concepts-en-acte, et ceux de Samurçay et Pastré (1995), qui mettent l'accent sur les concepts pragmatiques, amène la question suivante : en quoi les concepts pragmatiques se différencient-ils des concepts-en-acte ? Pour aborder cette question nous commencerons par préciser des caractéristiques communes aux concepts-en-acte et aux concepts pragmatiques, avant de présenter celles qui les différencient.

L'une des caractéristiques commune aux concepts-en-acte et aux concepts pragmatiques est qu'ils sont des « concepts » au sens que Vergnaud donne à ce terme, c'est-à-dire qu'ils sont définis par un triplet :

«-S : L'ensemble des situations donnant sens au concept.

— I : L'ensemble des invariants opératoires qui sont sous-jacents au traitement de ces situations par le sujet (le signifié).

— $\varsigma$ : L'ensemble des formes langagières et non langagières qui permettent de représenter le concept (le signifiant) $)^{21} \gg(1985$, p. 247-248; 1990, p. 145).

Prenons comme exemple les concepts pragmatiques de continuité et de sens de distribution de l'énergie, identifiés dans le domaine de la maintenance des systèmes électriques (Vidal-Gomel, 2001, 2007) :

On peut définir une classe générale de situations dans lesquelles ils sont mis en œuvre : les opérateurs doivent réaliser une mise hors tension pour travailler en sécurité sur un dispositif.

Continuité et sens de distribution de l'énergie sont des invariants opératoires qui sont au cœur de la représentation des réseaux électriques. Schématiquement, couper c'est rompre la continuité en amont du point à mettre hors tension, ce qui nécessite de se représenter l'ordre des éléments constitutifs du réseau (sens de distribution) et les relations de continuité entre ces éléments. De façon complémentaire, on a observé que les opérateurs décrivent une armoire électrique, par exemple, de l'amont vers l'aval (sens de distribution), en respectant la continuité entre les éléments.

Pour en parler, ils utilisent différentes expressions comme « amont / aval », « haut / bas », un disjoncteur «alimente» un dispositif, ou encore « remonter à l'armoire» pour rendre compte d'un déplacement de l'opérateur vers une armoire située en amont dans le réseau, mais sans que ce déplacement implique un tel mouvement pour l'opérateur : il «remonte » uniquement du point de vue de sa représentation du réseau.

Théorèmes-en-acte et concepts-en-acte ont à l'origine plutôt été identifiés dans le domaine de la didactique des disciplines, et plus particulièrement à propos de l'apprentissage des mathématiques, comme en témoignent d'ailleurs les exemples présentés plus haut. Les concepts pragmatiques concernent le domaine professionnel. Ce n'est pas ce qui fonde leur différence. Par exemple, pour implanter une cloison perpendiculaire à un mur, les maçons peuvent utiliser le théorème de Pythagore (Bessot, Deprez, Eberhard, \& Gomas, 1993).

Toutefois, un concept pragmatique n'est ni un concept-en-acte, ni un théorème-en-acte. Nous l'avons déjà souligné, les concepts pragmatiques sont verbalisés par les opérateurs. Ils sont transmis par les communautés professionnelles sous des formes langagières diverses. Nous en avons donné des exemples précédemment à propos des concepts pragmatiques de continuité et de sens de distribution de l'énergie. Par ailleurs, certaines variables observables ou mesurables liées au concept pragmatique peuvent être désignées, montrées. Pastré en donne un exemple dans ses travaux sur la conduite des presses à injecter : pour le concept pragmatique de bourrage, c'est « le mouvement présent ou absent de la partie mobile de la machine, au début de la phase statique » (1999b, p. 19).

Par ailleurs, les théorèmes-en-acte peuvent être vrais ou faux et, dans ce dernier cas, constituer un obstacle à l'apprentissage. De nombreux exemples existent dans la littérature concernant les ap-

$\overline{21 .}$ Notons que dans ce cadre les concepts ne sont pas uniquement des catégories. 
prentissages disciplinaires — par exemple, « les objets gardent la mémoire de leur passé » est une représentation constituant un obstacle à l'apprentissage de la physique, identifié par Viennot (1996). Les concepts pragmatiques ne peuvent pas être abordés de ce point de vue. Leur légitimité et leur pertinence sont essentiellement liées à l'efficacité de l'action (Rogalski, \& Samurçay, 1994 ; Rogalski, 2004).

Enfin, comme Vergnaud (1990) l'a précisé, théorèmes-en-acte et concepts-en-acte s'inscrivent dans des champs conceptuels. Ce n'est pas le cas des concepts dans un domaine professionnel. Les savoirs professionnels de référence ne comportent pas uniquement des concepts scientifiques et/ou techniques déterminés et connus (Rogalski, \& Samurçay, 1994). Pour en rendre compte, Pastré (1999b) utilise le terme de « champ professionnel».

\section{4.- Concepts pragmatiques, conceptualisation pour l'action et outils d'aide}

L'intérêt de la prise en compte des conceptualisations pour l'action - concepts pragmatiques ou, plus largement, structure conceptuelle des situations - dans un objectif de formation a été souligné dans plusieurs ouvrages récents (Pastré, 1999a ; Samurçay, \& Pastré, 2004 ; Pastré, 2005b). Nous souhaitons ici aborder un autre aspect : l'intérêt de leur prise en compte pour la conception d'outils d'aide (au diagnostic, à la décision, etc.).

Dans son bilan des caractéristiques de l'analyse du travail dans un objectif de conception écologique des artefacts, Vicente (1999) a souligné la nécessité de compatibilité entre les représentations des opérateurs et les artefacts conçus, tout en prenant en compte le fait que ces représentations puissent être en partie erronées. De notre point de vue, il s'agit de prendre en compte leurs conceptualisations.

Une autre orientation, située dans le cadre d'une approche instrumentale, consiste à prendre en compte les schèmes d'utilisation du sujet (Rabardel, 1995 ; Folcher, \& Rabardel, 2004). Nous retiendrons de cette approche le point de vue développemental dont elle est porteuse, ce qui conduit à intégrer la problématique du développement du sujet aux questions de conception, comme le proposent plusieurs auteurs (Falzon, 1996 ; Rabardel, \& Pastré, 2005). Toutefois, cette approche est centrée sur les genèses instrumentales et, si cette dimension des compétences n'est pas absente de notre propos, elle n'en est toutefois pas le centre.

En fait, en discutant de l'intégration de la structure conceptuelle des situations et des concepts pragmatiques à la conception des artefacts, nous nous intéresserons plus spécifiquement aux « outils cognitifs opératifs » (OCO) : ce sont des artefacts à visée instrumentale intégrant des savoirs de référence du domaine professionnel, de sorte que le sujet peut leur déléguer certaines opérations cognitives (Rogalski, \& Samurçay, 1993 ; Rogalski, \& Durey, 2004). L'utilisation des OCO peut constituer un moyen de favoriser le développement des compétences par « intériorisation des concepts incorporés dans l'outil [et/ou] intériorisation des schèmes sociaux d'utilisation » (Rogalski, \& Durey, p. 144).

Ainsi, comparativement aux instruments analysés par Rabardel (op. cit.), si les OCO sont des médiateurs entre le sujet et l'objet sur lequel il souhaite agir, cette médiation n'est pas directement pragmatique - elle ne concerne pas une transformation de cet objet, au niveau de l'agi —, ni épistémique - elle s'appuie sur des connaissances dont dispose déjà le sujet sur l'objet de l'action —, mais elle se situe au niveau des opérations cognitives effectuées sur des représentations, l'OCO étant un médiateur entre les représentations mentales liées à la conceptualisation du sujet et les représentations implémentées dans l'outil.

Nous nous appuierons sur trois exemples pour développer le rôle des concepts pragmatiques dans l'usage des OCO. Dans un premier temps, les travaux de Béguin (2005a, 2005b) sur la conduite d'un processus dynamique nous permettent de souligner, d'une part, qu'il s'agit de prendre en compte les 
conceptualisations des opérateurs pour concevoir un outil d'aide à la conduite et, d'autre part, qu'il peut s'avérer nécessaire d'amener les opérateurs à élargir leurs conceptualisations ${ }^{22}$ pour une utilisation complète de cet outil cognitif. Les travaux de Samurçay et Hoc dans le domaine de la conduite de haut-fourneau (Hoc, \& Samurçay, 1992 ; Samurçay, \& Hoc, 1996 ; Rabardel, \& Samurçay, 2001) nous fournissent un exemple d'outil conçu dans le but d'élargissement des conceptualisations des conducteurs (nous le dénommerons ici « outil HF »). Enfin, nous présenterons des résultats d'études d'un OCO conçu au sein d'une communauté professionnelle pour son propre usage : l'outil de conduite de la lutte contre des feux de forêt (« outil FF ») (Rogalski, \& Samurçay, 1993 ; Rogalski, \& Durey, 2004).

Ces résultats seront présentés en différenciant la syntaxe de l'outil (les représentations externes: courbes, etc.) et sa sémantique (les concepts pragmatiques incorporés dans l'outil) de façon à identifier différents problèmes de transparence opérative, c'est-à-dire « les propriétés caractéristiques de l'instrument pertinentes pour l'action de l'utilisateur, ainsi que la manière dont l'instrument les rend accessibles, compréhensibles, voire perceptibles pour l'utilisateur » (Rabardel, 1993, p. 109). Dans un dernier temps, nous discuterons de la conception des OCO dans un objectif de développement des compétences des opérateurs, en intégrant les apports de la différenciation syntaxe et sémantique et quelques questions que pose la transparence opérative.

\section{1.- Prendre en compte les conceptualisations pour l'action des opérateurs dans la conception des artefacts}

Les travaux de Béguin (2005a, 2005b) portent sur la conception d'un dispositif de sûreté (alarme) pour des sites classés SEVESO de la chimie fine. La décision de conception de cette alarme fait suite à une expertise juridique d'un accident mortel dû à un emballement de réaction. Pour prédire l'emballement de réaction, l'artefact conçu prélève des informations sur l'évolution de la température du produit et calcule un temps moyen restant avant une explosion (TMR). L'emballement de réaction est du côté du seuil haut des températures.

Dans un premier temps de l'étude, les analyses de l'activité réalisées révèlent que les opérateurs conduisent le process à partir du seuil bas des températures, "au plus près de la cristallisation » du produit. La cristallisation est leur référence, étant donné qu'il faut produire sans risque. La cristallisation est toutefois également un risque sérieux qu'il faut éviter et, pour les opérateurs, il faut gérer ce risque au quotidien. Aussi ils prélèvent différentes informations sur le produit : zébrures et marbrures, auxquelles correspondent les concepts de cristallisation et d'amorce de cristallisation, ils remplissent une feuille de conduite afin de disposer d'un indicateur sur l'évolution de la température pour pouvoir évaluer « la rapidité avec laquelle on s'approche du froid » (Béguin, 2005b).

Zébrures, marbrures et évolution de la température du produit semblent, de notre point de vue, constituer des indicateurs qui sont en relation avec les notions de cristallisation et d'amorce de cristallisation, concepts organisateurs ${ }^{23}$ de l'activité des opérateurs. Cet ensemble caractériserait - au moins en partie - le modèle opératif des opérateurs.

Dans un second temps, l'artefact conçu (l'alarme) est introduit dans la situation de travail. Une analyse de la direction des regards des opérateurs fait apparaitre qu'ils utilisent les informations sur la température, disponibles sur l'artefact, et non le TMR, qui constituait l'intérêt du dispositif étant donné les objectifs initiaux. La genèse instrumentale est ici caractérisée par une catachrèse : «les usagers ont attribué à l'alarme une fonction de thermomètre » souligne Béguin (2005a, p. 46). Le nouvel artefact leur fournit des informations plus précises sur l'évolution de la température que ce dont ils disposaient antérieurement.

On pourrait dire qu'ils ont ainsi pu affiner l'information relative à l'un de leurs indicateurs, et que

22. Dans l'optique de Béguin le développement est aussi bien celui des utilisateurs que celui des concepteurs. Notre propos est plus réduit : nous ne nous intéressons ici qu'aux utilisateurs.

23. Nous utilisons ici le terme de « concept organisateur » dans la mesure où il nous est difficile d'affirmer que la cristallisation ou l'amorce de cristallisation sont des concepts pragmatiques. 
l'usage de ce nouvel instrument ne modifie que très peu leur modèle opératif centré sur la cristallisation.

La non prise en compte des conceptualisations des opérateurs conduirait à un échec du projet. En effet, les informations sur l'emballement ne sont pas directement utiles aux opérateurs pour conduire le processus, puisqu'ils conduisent du côté de la cristallisation, et qu'ils n'ont pas pu acquérir en situation de connaissances à ce propos, étant donné les risques. Ainsi dans un troisième temps, la prise en compte des conceptualisations des opérateurs a permis une réorientation du projet de conception. Il s'agit d'outiller l'expertise dont disposent les opérateurs sur la cristallisation et de les amener à élargir leurs conceptualisations en y intégrant la problématique de l'emballement (Béguin, 2005a ; 2005b).

\section{2.- Concevoir des artefacts pour favoriser le développement des conceptualisations pour l'action}

L'outil HF est un outil d'aide à la conduite de haut-fourneau qui a été conçu ${ }^{24}$ avec l'objectif d'élargir les relations entre concepts pragmatiques et indicateurs pris en compte par les opérateurs (Hoc, \& Samurçay, 1992 ; Samurçay, \& Hoc 1996 ; Rabardel, \& Samurçay, 2001). Cet outil intègre l'ensemble des indicateurs nécessaires pour faire des inférences sur un concept pragmatique et les relations causales directes et indirectes entre concepts et indicateurs, contrairement à l'interface dont disposaient jusque-là les opérateurs, qui était organisée de façon topographique (décomposition du process en entités physiques).

La nouvelle interface conçue contraint la prise d'information : elle est hiérarchisée et pré-structurée par le modèle de relations causales sous-jacent et nécessite de générer des hypothèses. Les paramètres observables ou mesurables (indicateurs) sont regroupés autour des concepts pragmatiques qu'ils permettent d'évaluer. Ainsi, quand un opérateur veut consulter la valeur d'un paramètre, il doit rendre son choix d'un concept pragmatique explicite et s'appuyer sur les relations entre indicateurs et concepts, et entre concepts pragmatiques.

L'expérimentation est composée de deux sessions d'utilisation du nouvel outil, espacées d'un mois, par des professionnels de la conduite de haut-fourneau. Au cours de ces sessions, les opérateurs sont invités à traiter des situations de simulation avec cet outil. Entre ces deux sessions, l'outil est laissé en libre accès dans l'espace de travail.

Parmi les résultats les plus marquants (pour le détail voir Samurçay, \& Hoc, 1996), on relève, d'une session à l'autre, d'une part, que l'utilisation des indicateurs devient plus complète. Les opérateurs utilisent différents indicateurs pour confirmer ou infirmer une hypothèse. Les relations entre concepts pragmatiques et indicateurs se développent. D'autre part, davantage de concepts sont utilisés pour analyser la situation en cours. Les diagnostics sont de meilleure qualité et s'appuient sur la mise en relation de plusieurs concepts. Ainsi les relations entre les concepts pragmatiques semblent mieux établies et les opérateurs semblent s'être approprié le modèle comme un tout permettant d'analyser la situation en cours, et d'envisager les actions à mener (Samurçay, \& Hoc, 1996 ; Rabardel, \& Samurçay, 2001). Toutefois, il faut noter que les résultats sont plus décevants concernant les activités de pronostic - anticipation des états futurs possibles du process - ou les activités de prise de décision — anticipation des actions pour prévenir des dérives (Samurçay, \& Hoc, 1996).

\section{3.- Sémantique, syntaxe et transparence opérative d'un OCO}

L'exemple considéré est celui de l'utilisation de l'outil « feu de forêt» (FF). Cet outil a été conçu dans un objectif d'aide à la décision, au niveau tactique, dans le domaine de la lutte contre les feux de forêt (Rogalski \& Durey, 2004). Dans ce domaine professionnel, et à ce niveau de décision, il s'agit d'élaborer la «solution du feu» qui est déterminée par «l'équation du feu»: «les moyens qu'il

24. Le processus de conception de l'artefact a intégré la participation des ingénieurs et des opérateurs. 
faut, à l'endroit qu'il faut, au moment où il faut, pour faire ce qu'il faut» (op. cit., p. 116).

L'outil FF est un outil « représentatif », qui intègre à la fois « un modèle de la dynamique du feu dans son espace de propagation et un modèle des moyens d'action » (op. cit., p. 112). Il permet de se représenter le feu (l'origine, l'axe de propagation du feu et la zone menacée) et la «balance des moyens » (comparaison des moyens nécessaires pour stopper le feu et des moyens disponibles, en fonction du temps). Des concepts pragmatiques du domaine sont sous-jacents à ces représentations : axe de propagation, front, vitesse de propagation du feu, et « équivalent engins » (ou « engins théoriques »).

Une expérimentation a été réalisée avec des officiers sapeurs-pompiers, ayant eu ou non une formation à l'utilisation de l'outil FF et ayant ou pas une expérience des feux de forêt (Rogalski, \& Durey, op. cit.). Les résultats sont interprétés en différenciant la syntaxe de l'outil (les représentations externes : courbes, etc.), et sa sémantique (les concepts pragmatiques incorporés dans l'outil). Dans ce cadre, trois cas de relations à la transparence opérative sont observés :

- Syntaxe et sémantique sont totalement transparentes. Quelles que soient leur expérience et leur formation, les officiers sont en mesure d'identifier les renforts à demander pour une action précise en utilisant les courbes du feu et les courbes des moyens proposées par l'outil. Dans ce cas, syntaxe et sémantique ne nécessitent que des schèmes et des conceptualisations répandus, partagés dans le domaine professionnel.

- Un format syntaxique atypique par rapport aux schèmes des opérateurs constitue un obstacle à l'utilisation de l'outil. Ainsi, les représentations externes spontanées de l'évolution du feu sont des représentations analogiques prenant en compte l'espace topographique dans lequel se développe le feu. Les opérateurs tracent spontanément des courbes pour indiquer l'évolution du feu à t0, t1, t2, etc. En revanche, la syntaxe de l'artefact est composée d'un espace bidimensionnel abstrait (espace $\mathrm{x}$ temps), qui permet de représenter l'évolution du feu par une droite, en faisant l'hypothèse que sa vitesse est constante. L'écart entre ces deux formes de représentations externes est important. Pour les officiers, ce que l'on peut ou non représenter en fonction de la syntaxe de l'artefact constitue une réelle difficulté, mais une initiation à l'outil FF permet une acquisition rapide, dans la mesure où les représentations fonctionnelles requises reposent sur des acquis scolaires antérieurs.

- La sémantique de l'outil peut poser problème dans des tâches de prévision ${ }^{25}$. Par exemple, l'une des difficultés des opérateurs relève du concept pragmatique d'engin théorique.

L'utilisation courante de l'outil FF ne nécessite pas ce concept, en revanche il est requis pour traiter certains types de problèmes. Ainsi, quand on utilise une diversité de moyens dont des engins aériens, il est nécessaire de rechercher un « équivalent engin ».

Le concept d' « engin théorique » est évalué à partir de différents paramètres, comme, par exemple, le débit d'eau, le volume d'eau que peut embarquer un engin, le volume réellement pris, ce qui est renouvelé, le temps nécessaire pour ce renouvellement, etc. Ces paramètres sont eux-mêmes évalués à partir de différentes variables. Par exemple, à proximité de la mer, il vaut mieux disposer de Canadairs que de Trackers. Le temps de renouvellement - aller faire le plein et revenir sur le lieu de l'opération - est de deux à trois fois plus court. Deux variables principales interviennent dans le calcul : le volume d'eau disponible et son débit. Le temps de renouvellement du potentiel en eau constitue une variable intermédiaire. Ainsi la difficulté de ce concept tient au nombre de paramètres à intégrer et au fait que ces paramètres sont des variables construites et intermédiaires, qui doivent être évaluées à partir d'autres variables. Ces caractéristiques, qui sont partagées par de nombreux concepts pragmatiques que nous avons présentés (par exemple concernant la taille de la vigne ou la conduite de hauts-fourneaux), sont sans doute des indicateurs de la complexité des tâches d'un domaine. Le fonctionnement le plus fréquemment observé dans l'étude (Ibid.) est d'identifier « engins théoriques » à « engins réels » : les opérateurs ne maîtrisent pas le concept pragmatique. Nous

25. Le terme « prévision » a ici un sens professionnel : il s'agit non pas d'une anticipation de l'évolution d'un sinistre en cours, mais de prévoir une possible future intervention sur un sinistre. Cette intervention future potentielle requiert de traiter des variables touchant aussi bien des propriétés du sinistre (ici la vitesse du feu en fonction de la vitesse du vent et de l'hygrométrie) que des propriétés des moyens d'intervention (ici établir une correspondance entre des " équivalents engins » et des moyens réels, différant en nature et en positionnement par rapport au sinistre). 
avons également observé, dans une tâche de prévision d'évolution du feu, une conception erronée de l'importance respective de la vitesse du vent et de l'hygrométrie sur la vitesse du feu, existant chez beaucoup de sujets y compris quand ils avaient une expérience du feu de forêt ou une formation à l'outil ; conception erronée alors ignorée des formateurs. L'utilisation de l'outil FF dans des tâches de prévision permet donc d'identifier deux types de difficultés.

L'analyse de l'utilisation de l'outil FF en différenciant syntaxe et sémantique révèle donc des problèmes de conception - la syntaxe n'est pas transparente quand il s'agit de s'intéresser à l'évolution du feu - et des besoins de formation.

\section{4.- Concevoir un outil d'aide avec un objectif de développement des compétences des opérateurs}

Les trois études précédemment présentées nous permettent de souligner quelques caractéristiques de la conception des artefacts dans un objectif de développement des compétences des opérateurs.

Nous avons mis l'accent sur l'intérêt d'intégrer aux artefacts les concepts pragmatiques du domaine. Les travaux de Béguin (2005a, 2005b) nous semblent souligner qu'au-delà des représentations des opérateurs, il est nécessaire de prendre en compte leurs conceptualisations. Dans ce cadre, concevoir des outils cognitifs opératifs en intégrant la structure conceptuelle des situations permet de s'assurer de la compatibilité avec les modèles des opérateurs, tout en répondant à des critères de véracité et de pertinence au regard des caractéristiques et propriétés de l'objet de l'action professionnelle. L'identification de la structure conceptuelle des situations relève en effet de la mise en évidence de « savoirs professionnels de référence » - « catégories d'objets et de traitements communes aux pratiques efficaces » (Rogalski, \& Samurçay, 1994, p. 43). Ainsi, comparativement à l'approche de conception écologique de Vicente et Rasmussen (Vicente, 1999), l'intégration des concepts pragmatiques dans les outils prend en compte les représentations des opérateurs, tout en répondant aux exigences de pertinence, nécessité soulignée par Vicente.

Par ailleurs, différencier la syntaxe et la sémantique d'un artefact conduit à réinterroger la notion de transparence opérative. Schématiquement, une transparence maximale renvoie à un artefact dont la sémantique et la syntaxe nécessitent des conceptualisations et des schèmes communs dans un domaine professionnel. Un tel artefact est adapté à la dimension productive de l'activité mais peu à sa dimension constructive, telle qu'elle est définie par Samurçay et Rabardel (2004). Cela peut s'avérer utile pour des débutants dans un métier, mais des professionnels expérimentés vont pouvoir fonctionner sur la base de leurs routines. Autrement dit, ils apprendront peu, voire rien.

En même temps, la transparence opérative reste un critère important quand il s'agit de concevoir avec un objectif de développement. Nous avons, en effet, a contrario vu l'exemple d'un artefact dont la syntaxe, atypique au regard des schèmes des opérateurs, devient un obstacle à l'utilisation de l'outil.

S'adressant à des professionnels, une première voie de conception consiste alors à ne pas rendre la syntaxe complètement transparente pour faire travailler la sémantique. L'outil HF a été conçu avec l'objectif d'élargir le réseau de relations entre variables, concepts pragmatiques et indicateurs dont disposent les opérateurs. En effet, la sémantique de l'outil est constituée à partir du réseau de relations utilisé et connu des opérateurs et enrichi par un travail réalisé auprès des ingénieurs du domaine (Samurçay, 1995). La syntaxe est conçue de façon à obliger les opérateurs à élaborer des hypothèses sur le process conduit et son évolution, et à les rendre explicites pour accéder aux informations recherchées. Autrement dit, dans ce cas, la syntaxe oblige à utiliser plus explicitement la structure conceptuelle de la situation (sémantique), ce qui a constitué un facteur de développement (Hoc, \& Samurçay, 1992 ; Samurçay, \& Hoc 1996 ; Rabardel, \& Samurçay, 2001).

Une orientation inverse est utilisée par Boucheix dans ses travaux sur les grutiers (Boucheix, \& Chanteclair, 1999 ; Boucheix, 2003). Il utilise les conceptualisations acquises dans l'action par ces opérateurs - la sémantique qui est ici notamment composée des relations «poids levé x portée » et 
des indicateurs utilisés par les opérateurs (klaxon, limites de vitesse, coupe-circuit) - et une représentation externe analogique pour les former à la lecture des courbes de limites de transport des charges des grues ; la formation vise l'acquisition de cette syntaxe des relations «poids x portée ». L'impossibilité de lire et d'interpréter spontanément ces courbes des charges constituaient en effet pour ces grutiers expérimentés, souvent peu lettrés ou illettrés, un obstacle important à la réussite de leur examen de certification. Ainsi, on peut dire que Boucheix s'appuie sur la sémantique constituée par les opérateurs pour leur faire acquérir une syntaxe particulière (les courbes des charges), en utilisant une représentation externe analogique, syntaxe intermédiaire, qui va fonctionner comme un «pont cognitif », pour reprendre les termes de l'auteur (Boucheix, 2003).

Enfin, les travaux de Rogalski et Durey (2004) montrent que différentes tâches utilisant l'outil FF ne requièrent pas la même profondeur d'intégration par le sujet de la syntaxe de l'outil et de sa sémantique. On peut jouer de ces décalages éventuels dans un objectif de développement des compétences : ainsi, des officiers non familiers avec les feux de forêt, mais formés à l'utilisation de la syntaxe des courbes ont eu accès à des propriétés de l'action sur le feu, dans des tâches simples; à l'inverse, dans l'étude de Boucheix (op. cit.), les grutiers, par le biais de situations de simulation mobilisant les compétences qu'ils ont acquises dans des tâches familières, ont pu accéder à l'utilisation d'une syntaxe qui peut se révéler longue à acquérir dans le champ de l'éducation scolaire. Mais l'utilisation de l'outil n'est pas garante de l'acquisition des concepts nécessaires à la réalisation de certaines tâches. L'échec dans le traitement de tâches de prévision exigeantes en matière sémantique peut rendre explicite des limites de la conceptualisation, et des besoins de formation correspondant.

De ces travaux, quelques lignes de réflexion peuvent être dégagées. Pour concevoir des outils avec un objectif développemental, il est possible, d'une part, d'intégrer les concepts pragmatiques et/ou la structure conceptuelle des situations à l'artefact, d'autre part, de jouer sur la syntaxe de l'artefact pour faire acquérir par l'action la sémantique, ou inversement. Ces questions doivent être appréhendées en mettant en relation transparence opérative de l'artefact et zone proximale de développement des opérateurs (Vygotski, 1934/1997). Les outils cognitifs opératifs peuvent aussi être utilisés pour identifier des besoins de formation en jouant sur la complexité des tâches à réaliser, puisque certaines tâches nécessitant une plus grande et plus complète maîtrise de la sémantique de l'outil constituent de bons révélateurs des limites de la conceptualisation des opérateurs.

Pour conclure, dans cet article, nous avons voulu discuter d'un concept central dans la théorie de la didactique professionnelle, développée en France à partir de la conjonction de travaux de psychologie ergonomique et de didactique disciplinaire, celui de « concept pragmatique ».

La mise en perspective historique de ce concept a permis de donner des exemples dans la littérature montrant que les concepts pragmatiques formaient le noyau d'une «structure conceptuelle de la situation ", exprimant des relations de détermination causale entre concepts, et des liens avec les indicateurs de prise d'information ou de commande d'action. Cette structure conceptuelle est le résultat d'un processus de conceptualisation, permettant aux acteurs une représentation mentale de la situation, orientée vers l'action. Ce processus est socio-historique, au sens où il se déroule dans le temps long du développement du collectif de travail - en interaction éventuellement avec une analyse ergonomique ou didactique du travail —, et où tout nouvel acteur, pour développer sa compétence professionnelle, doit intérioriser et donner du sens aux concepts pragmatiques qui sont agis et exprimés dans le travail.

Nous avons situé cette notion propre à la didactique professionnelle par rapport à des concepts d'autres cadres théoriques qui s'intéressent à l'organisation des connaissances et à la construction de représentations ou de modèles mentaux, en soulignant particulièrement deux points : d'une part, un concept pragmatique est une construction mentale dont les propriétés l'apparentent aux concepts techniques ou scientifiques mais, à la différence de ceux-ci, son domaine de validité est précisément circonscrit à une catégorie de situations de travail dans un domaine professionnel précis, et la pierre de touche de cette construction n'est pas la validation théorique mais l'efficacité pratique. D'autre part, si un concept pragmatique a un caractère adaptatif à l'action tout comme les concepts quotidiens ou les concepts-en-acte, il s'intègre dans une représentation cohérente (la structure conceptuelle de 
la situation) et il est explicité dans la communication dans le cours du travail (il y a toujours « des mots pour le dire »).

Les travaux publiés en didactique professionnelle donnent de multiples exemples de l'importance de la notion de concept pragmatique pour analyser les compétences et leur formation. Sans y revenir, nous avons choisi de montrer, sur quelques exemples, la pertinence de cette notion pour la conception d'outils cognitifs.

\section{RÉFÉRENCEMENT}

Vidal-Gomel, Ch., \& Rogalski, J. (2007). La conceptualisation et la place des concepts pragmatiques dans l'activité professionnelle et le développement des compétences. @ctivités, 4 (1), pp 49-84, http://www. activites.org/v4n1/v4n1.pdf

\section{RÉFÉRENCES}

Anderson, J.R. (1983). The architecture of cognition. Cambridge: Harvard University Press.

Anderson, J.R. (1987). Skill acquisition: compilation of weak-method problem solutions. Psychological Review, 94, 192-210.

Anderson, J.R., \& Fincham, J.M. (1994). Acquisition of procedural skills from examples. Journal of Experimental Psychology: Learning Memory and Cognition, 20, 1322-1340.

Argyris, C., \& Schön D.A. (1978). Organisational learning. A theory of action perspective. Readling: Addison-Wesley.

Argyris, C., \& Schön D.A. (1996/2002). Apprentissage organisationnel. Théorie, méthode, pratique. Paris: DeBoeck Université.

Bainbridge, L. (1988). Different representations, and the implications for cognitive processing. In L.P. Goodstein, H.B. Anderson, \& S.E. Olsen (Eds.), Tasks, errors and mental models (pp. 70-91). London: Taylor and Francis Ltd.

Béguin, P. (2005a). Concevoir pour les genèses professionnelles. In P. Rabardel, \& P. Pastré (Eds.), Modèles du sujet pour la conception. Dialectiques activités développement (pp. 31-52). Toulouse: Octarès.

Béguin, P. (2005b). La simulation entre experts : double jeu dans la zone proximale de développement et construction de mondes communs. In P. Pastré (Ed.), Apprendre par la simulation. De l'analyse du travail aux apprentissages professionnels (pp. 55-77). Toulouse: Octarès.

Bessot, A., Déprez, S., Eberhard, M., \& Gomas, B. (1993). Une approche didactique de la lecture de graphismes techniques en formation professionnelle de base aux métiers du bâtiment. In A. Bessot, \& P. Vérillon (Eds), Espace graphique et graphismes d'espace. Contribution de psychologues et de didacticiens à l'étude de la construction des savoirs spatiaux (pp. 115-144). Grenoble: La Pensée Sauvage.

Bisseret, A., \& Enard, C. (1969-1970). Problème de la structuration de l'apprentissage de l'apprentissage d'un travail complexe : une méthode de formation par interaction constantes des unités programmées. Bulletin de Psychologie, 23, 632-648.

Boreham, N. (2002). Work process knowledge in technological and organizational development. In N. Boreham, R. Samurçay, \& M. Fischer (Eds.), Work process knowledge (pp. 1-14). London: Routledge.

Boreham, N., Samurçay, R., \& Fischer, M. (2002) (Éds.). Work process knowledge. London : Routledge.

Boshuizen, H.P.A., Schmidt, H.G., Custers, J.F.M., \& Van de Wiel, M.W. (1995). Knowledge development and restructuring in the domaine of medicine: the role of theory and practice. Learning and Instruction, 24, 4, 269-289.

Boucheix, J.-M. (2003). Simulation et compréhension de documents techniques : le cas de la formation des grutiers. Le Travail Humain, 66, 3, 255-287.

Boucheix, J.-M., \& Chanteclair, A. (1999). Analyse de l'activité, cognition et construction de situations d'apprentissage : le cas des conducteurs de grues à tours. Éducation permanente, 139, 115-141. 
Boutinet, J.-P. (2004). Vie adulte et formation permanente : de la notion au concept. In P. Carré, \& P. Caspar (Eds.). Traité des sciences et des techniques de la formation (pp. 199-218). Paris: Dunod (2édition).

Buty, C., \& Cornuéjols, A. (2002). Évolution des connaissances chez l'apprenant. In A. Tiberghien (Éd.), Des connaissances naïves aux savoirs scientifiques (pp. 41-66). Rapport de synthèse commandé par le programme « École et sciences cognitives » (mars, 2002).

Caens-Martin, S. (2005). Concevoir un simulateur pour apprendre à gérer un système vivant à des fins de production : la taille de la vigne. In P. Pastré (Ed.), Apprendre par la simulation. De l'analyse du travail aux apprentissages professionnels (pp. 81-106). Toulouse: Octarès.

Carey, S. (1985). Conceptual change in childwood. Cambridge (MA): MIT Press.

Carré, P., \& Caspar, P. (2004) (Eds.). Traité des sciences et des techniques de la formation. Paris: Dunod ( $2^{\mathrm{e}}$ édition).

Chevallard, Y. (1985/1991). La transposition didactique. Grenoble: La pensée sauvage.

Chi, M.T.H. (2005). Commonsense conceptions of emergent processes : why some misconceptions are robust. The Journal of the Learning Sciences, 14 (2), 161-199.

Closset, J.-L. (1989). Les obstacles à l'apprentissage de l'électrocinétique. Bulletin de l'Union des Physiciens, 716, 931-948.

Constant, E.W. (1980). The origins of the turbojet revolution. Baltimore: Johns Hopkins University Press.

Doane, S, Pellegrino, J, \& Klatsky, R L. (1990). Expertise in a computer operating system: Conceptualization and performance. Human-Computer Interaction, 5(2), 267-304

Dugrillon, D. (2002). Opérer en sécurité sur un ouvrage électrique. Incendie sur un ouvrage électrique (fascicule de documentation $\mathrm{n}^{\circ} 5,3^{\text {ème }}$ édition). Risques professionnels, la ligne prévention CRAM Centre (en ligne : http://www.cram-centre.fr, consulté en mai 2005).

Engeström, Y. (1987). Learning by expanding: an activity-theoretical approach to developmental research. Helsinki: Orienta-Konsultit.

Engeström, Y. (1994). Training for change : new approach to instruction and learning in work life. Geneva: International labour office.

Falzon, P. (1996). Des objectifs de l'ergonomie. In F. Daniellou (Ed.), L'ergonomie et quête de ses principes (pp. 233-242). Toulouse: Octarès.

Falzon, P., \& Teiger, C. (2004). Ergonomie et formation. In P. Carré, \& P. Caspar (Eds.). Traité des sciences et des techniques de la formation (pp. 145-161). Paris: Dunod ( $2^{\mathrm{e}}$ édition).

Fischer, M., \& Boreham, N. (2004). Work process knowledge : origins of the concepts and current development. In M. Fischer, N. Boreham, \& B. Nyham (Eds.), European perspective on learning at work : the acquisition of work process knowledge (pp. 12-53). Luxembourg: Office for official publications of the european communities.

Fischer, M., Boreham, N., \& Nyham, B. (Eds.) (2004). European perspective on learning at work: the acquisition of work process knowledge. Luxembourg: Office for official publications of the european communities.

Folcher, V., \& Rabardel, R. (2004). Hommes, artefact, activités : perspective instrumentale. In P. Falzon (Ed.), Ergonomie (pp. 251-268). Paris: PUF.

Gibbons, A.S. (2000). The practice of instructional psychology. Paper presented at AECT 2000 : annual international conference at the association for eductional communication and technology. Denver, october 2000 .

Ginsbourger, F., Merle, V., \& Vergnaud, G. (1992) (Eds.) Formation et apprentissage des adultes peu qualifiés. Paris: La documentation française.

Guide de la photographie panoramique. (en ligne : http://www.arnaudfrichphoto.com/, consulté le 9/12/05).

Hahn, C. (1999). Proportionnalité et pourcentages chez les apprentis vendeurs. Réflexion sur la relation mathématique/réalité dans une formation en «alternance ». Educational studies in mathematics, 39, 229249. 
Harvey, L. \& Anderson, J.R. (1996). Transfert of decalrative knowledge in complex information processing domains. Human-Computers Interaction, 11, 69-96.

Hoc, J.-M. (1989). La conduite d'un processus à longs délais de réponse : une activité de diagnostic. Le Travail Humain, 52, 4, 289-316.

Hoc, J.-M. (1996). Supervision et contrôle de processus. La cognition en situation dynamique. Grenoble: PUG.

Hoc, J.-M., \& Samurçay, R. (1992). An ergonomic approach to knowledge representation. Reliability Engenieering and System Safety, 36, 217-230.

Joshua, S., \& Dupin, J.-J. (1993). Introduction à la didactique des sciences et des mathématiques. Paris: PUF.

Kaufman, D.R. (2001).

Scientific explanation, systematicity, and conceptual change. In CogSci2000 : Twenty second annual meeting of the cognitive science society. Philadelphia, Pennsylvania, USA (13-15 août 2000) (en ligne : http://www.ircs.upenn.edu/ ircs/cogsci2000/symposia.shtml).

La correction de la parallaxe (en ligne : http://www.summilux.net/documents/parallaxe.html, site consulté le 12/12/2005).

Lave, J., \& Wenger, E. (1991). Situated learning. Legitimate peripheral participation. Cambridge: Cambridge university press.

Leplat, J. (2002). Psychologie de la formation. Jalons et perspectives. Choix de textes (1955-2002). Toulouse: Octarès.

Mayen, P., \& Savoyant, A. (1999). Application de procédures et compétences. Formation Emploi, 67, 77-92.

Norman, D. A. (1983). Some observations on mental models. In D.R. Gentner, \& A.L. Stevens (Eds.), Mental models (pp. 7-14). Mahwah, NJ: Lawrence Erlbaum Associates.

Ochanine, D. (1978). Le rôle des images opératives dans la régulation des activités de travail. Psychologie et Éducation, 2, 63-72.

Pastré, P. (1992a). Requalification des ouvriers spécialisés et didactique professionnelle. Éducation permanente, 111, 33-53.

Pastré, P. (1992b). Apprentissage sur le tas et conceptualisation dans la conduite de machines automatisées. In F. Ginsbourger, V. Merle, \& G. Vergnaud, G. (Eds.), Formation et apprentissage des adultes peu qualifiés (pp. 205-209). Paris: La documentation française.

Pastré, P. (1994). Variations sur le développement des adultes et leurs représentations. Éducation permanente, 119, 33-63.

Pastré, P. (1997). Didactique professionnelle et développement. Psychologie française, 42(1), 89-100.

Pastré, P. (1999a) (Ed.). Éducation permanente, 139, numéro spécial : Apprendre des situations.

Pastré, P. (1999b). La conceptualisation dans l'action : bilan et nouvelles perspectives. Éducation permanente, 139, 13-35.

Pastré, P. (2004a). Le rôle des concepts pragmatiques dans la gestion des situations problèmes : le cas des régleurs en plasturgie. In R. Samurçay, \& P. Pastré (Eds.), Recherches en didactique professionnelle (pp. 17-47). Toulouse: Octarès.

Pastré, P. (2004b). L’ingénierie didactique professionnelle. In P. Carré, \& P. Caspar (Eds.). Traité des sciences

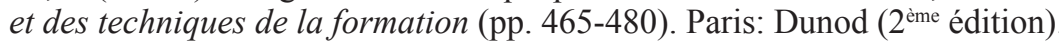

Pastré, P. (2005a). La conception de situations didactiques à la lumière de la théorie de la conceptualisation dans l'action. In P. Rabardel, \& P. Pastré (Eds.), Modèles du sujet pour la conception. Dialectiques activité développement (pp. 73-107). Toulouse: Octarès.

Pastré, P. (2005b) (Ed.). Apprendre par la simulation. De l'analyse du travail aux apprentissages professionnels. Toulouse: Octarès.

Pastré, P., Mayen, P., \& Vergnaud, G. (2006). La didactique professionnelle. Revue Française de Pédagogie, 154, 1-55. 
Patel, V.L., Evans, D.A., \& Groen, G.J. (1989). Biomedical knowledge and clinical reasonning. In D.A. Evans, \& V.L. Patel (Eds.), Cognitive science in medecine: Biomedical modelling (pp. 53-112). Cambridge (MA): MIT Press.

Patel, V.L., \& Kaufman, D.R. (1995). Clinical reasoning and biomedical knowledge : implications for teaching. In J. Higgs, \& M. Jones (Eds.), Clinical reasonning in the health professions (pp. 117-128). Oxford (UK): Butterwoorth-Heinnemann.

Patel, V.L., Kaufman, D.R., \& Anocha, J.F. (2002). Emerging paradigms of cognition in medicine decision making. Journal of Biomedical Informatics, 35(1), 52-75.

Patrick, J. (1992). Training research and practice. London: Academic press.

Percier, M. (1990). Guide de conduite. Nantes: ENMM, département simulateur machine.

Piaget, J. (1974). La prise de conscience. Paris: PUF.

Pineau, G. (2004). Expériences d'apprentissage et histoires de vie. In P. Carré, \& P. Caspar (Ed.), Traité des sciences et des techniques de la formation (pp. 319-335). Paris: Dunod.

Poitrenaud, S. (1995). The Procope semantic network: an alternative to action grammars. International Journal of Human-Computer Studies, 42, 31-69.

Poitrenaud, S. (2001). Complexité cognitive des interactions homme-machine. Paris: L'Harmattan.

Rabardel, P. (1993). Représentations dans les situations d'activités instrumentées. In A. Weill-Fassina, P. Rabardel, \& D. Dubois (Eds.), Les représentations pour l'action (pp. 97-111). Toulouse: Octarès.

Rabardel, P. (1995). Les hommes et les technologies, approche cognitive des instruments contemporains. Paris: Armand Colin.

Rabardel, P., \& Pastré, P. (Eds.) (2005). Modèles du sujet pour la conception. Toulouse: Octarès.

Rabardel, P., \& Samurçay, R. (2001). From artifact to instrument-mediated learning. Conférence invitée au symposium : New challenges to research on learning. Center of Activity theory and Developmental Work Research, Université d'Helsinki, 21-23 mars 2001 (en ligne : http://ergoserv.psy.univ-paris8.fr).

Randel, J.M., Pugh, H.L., \& Reed, S.K (1996). Differences in expert and novice situation awareness in naturalistic decision making. International Journal of Human-Computer Studies, 45(5), 579-597.

Richard, J.-F. (1983). Logique de fonctionnement et logique de l'utilisation. Rapport de recherche $\mathrm{n}^{\circ} 202$. INRIA, Rocquencourt.

Richard, J.-F. (2004). Les activités mentales. De l'interprétation de l'information à l'action. Paris: Armand Colin ( $4^{\mathrm{e}}$ édition refondue).

Rogalski, J. (1995). From real situations to training situations: concervation of functionnalities. In J.M. Hoc, P.C. Cacciabue, \& E. Hollnagel (Eds.), Expertise and technology: cognition and human-computer cooperation (pp. 123-137). Hillsdale (NJ): Lawrence Erlbaum Associates.

Rogalski, J. (2004). La didactique professionnelle : une alternative aux approches de « cognition située » et « cognitiviste» en psychologie des acquisitions. @ctivites, 1 (2). (en ligne : http://www.activites.org/).

Rogalski, J. (2006). Articulation des théories de Piaget et de Vygotsky, outils pour la didactique. In C. Castela, \& C. Houdement (Eds.), Actes du séminaire national de didactique des mathématiques. Année 2005 (pp. 237-262). Paris: ARDM et IREM -PARIS 7.

Rogalski, J., \& Durey, A. (2004). Compétences, savoirs de références et outils cognitifs opératifs. In R. Samurçay, \& P. Pastré (Eds.), Recherches en didactique professionnelle (pp. 109-136). Toulouse: Octarès.

Rogalski, J., \& Marquié, J.-C. (2004). Évolution des compétences et des performances. In J.-M. Hoc, \& F. Darses (Eds.), Psychologie ergonomique : tendances actuelles (pp. 141-173). Paris: PUF.

Rogalski, J., \& Samurçay, R. (1993). Représentation de référence : outil pour la conduite d'environnement dynamiques. In A. Weill-Fassina, P. Rabardel, \& D. Dubois (Eds.), Les représentations pour l'action (pp. 183-207). Toulouse: Octarès.

Rogalski, J., \& Samurçay, R. (1994). Modélisation d'un savoir de référence et transposition didactique dans la formation de professionnels de haut niveau. In G. Arsac, Y. Chevalard, J.-L. Martinand, \& A. Tiberghien (Eds.), La transposition didactique à l'épreuve (pp. 35-71). Grenoble: La pensée sauvage. 
Rogalski, J., \& Veillard, L. (2002). Articulation entre différents types de connaissances. In A. Tiberghien (Ed.), Des connaissances naïves aux savoirs scientifiques (pp. 67-106). Rapport de synthèse commandé par le programme « École et sciences cognitives » (mars 2002).

Samurçay, R. (1995). Conceptual models for training. In J.M. Hoc, P.C. Cacciabue, \& E. Hollnagel (Eds.), Expertise and technology: cognition and human-computer cooperation (pp. 107-124). Hillsdale, NJ: Lawrence Erlbaum Associates.

Samurçay, R., \& Hoc, J.-M. (1988). De l'analyse du travail à la spécification d'aide à la décision dans les environnements dynamiques. Psychologie Française, 33(3), 187-196.

Samurçay, R., \& Hoc, J.-M. (1996). Causal versus topographical support for diagnosis in a dynamic situation. Le Travail Humain, 59(1), 45-68.

Samurçay, R., \& Pastré, P. (1995). La conceptualisation des situations de travail dans la formation des compétences. Education permanente, 123, 13-31.

Samurçay, R., \& Pastré, P. (Eds.) (2004). Recherches en didactique professionnelle. Toulouse: Octarès.

Samurçay, R., \& Rabardel, P. (2004). Modèles pour l'analyse de l'activité et des compétences, propositions. In R. Samurçay, \& P. Pastré (Eds.), Recherches en didactique professionnelle (pp. 163-180). Toulouse: Octarès.

Samurçay, R., \& Rogalski, J. (1992). Formation aux activités de gestion d'environnements dynamiques : concepts et méthodes, Éducation permanente, 111, 227-242.

Schmidt, H.G., \& Boshuizen, H.P.A. (1993). On acquiring expertise in medicine. Educational Psychology Rewiev, 5(3), 205-221.

Schmidt, H.G., Boshuizen, H.P.A., \& Hobus, P.P.M. (1988). Transitoiry stages in the development of medical expertise : the « intermediate effect» in clinical case representation studies. In Proceedings of the cognitive science society meeting. Hillsdale (NJ): Lawrence Erlbaum.

Sigaut, F. (1991). Les points de vue constitutifs d'une science des techniques. Essai de tableau comparatif. In J. Perrin (Ed.), Construire une science des techniques (pp. 31-45). Limonest: L'interdisciplinaire.

Thagard, P., \& Toombs, E. (2005). Atoms, categorization and conceptual change. In H. Cohen, \& C. Lefebvre (Eds.), Handbook of categorization in cognitive science (pp. 243-254). Amsterdam: Elsevier.

Tiberghien, A. (2002) (Éd.). Des connaissances naïves au savoir scientifique. Rapport de synthèse commandé par le programme « École et sciences cognitives » (mars 2002).

Tijus, C., Cambon de Lavalette, B., Poitrenaud, S., \& Leproux, C. (2003). L’interaction autorégulatrice entre dispositif et utilisateur : une modélisation des interférences sur les durées du parcours routier. Le Travail Humain, 66(1), 23-44.

Tout pour la science. Index de physique (en ligne : http://science-energie.chez-alice.fr/Physique/Parallaxe.

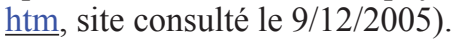

Tynjälä, P., Nuutinen, A., Eteläpleto, A., Kirjonen, J., \& Remes, P. (1997). The acquisition of professional expertise - a challenge for eductional research. Scandinavian Journal of Educational Research, 41 (3-4), 475-494.

Van de Wiel, M.W.J., Boshuizen, H.P.A., \& Schmidt, H.G. (2000). Knowledge restructuring in expertise development: evidence from pathophysiological representations of clinical cases by students and physicians. European Journal of Cognitive Psychology, 12, 3, 323-355.

Vergnaud, G. (1985). Concepts et schèmes dans une théorie opératoire de la représentation. Psychologie française, 30 (3-4), 245-251.

Vergnaud, G. (1990). La théorie des champs conceptuels. Recherches en didactique des mathématiques, 10(23), 133-170.

Vergnaud, G. (2004). Le développement cognitif de l'adulte. In P. Carré, \& P. Caspar (Eds.). Traité des sciences et des techniques de la formation (pp. 219-233) Paris: Dunod ( $2^{\mathrm{e}}$ édition).

Vérillon, P. (2002). Problème et technologie : brève incursion dans la littérature pour introduire le séminaire. In Y. Cartonnet, J. Lebeaume, \& P. Vérillon (Eds.), Problème et technologie. Actes du séminaire de didactique des disciplines technologiques Cachan 2000-2001 (pp. 9-22). Paris: Association Tour 123. 
Vicente, K. (1999). Cognitive work analysis. Towards safe, productive and healthy computer-based work. Mahwah (NJ): Lawrence Erlbaum Associates.

Vidal-Gomel, C. (2001). Le développement des compétences pour la gestion des risques professionnels. Le domaine de la maintenance des systèmes électrique. Thèse de psychologie ergonomique. Saint-Denis: Université Paris 8.

Vidal-Gomel, C. (2007, sous presse). Compétences pour gérer les risques professionnels : un exemple dans le domaine de la maintenance des systèmes électriques. Le travail humain, 70, 2.

Vidal-Gomel, C., Olry, P., Jeanmougin, H., \& Lanoë, D. (2005). Didactique professionnelle et gestion des risques professionnels : l'exemple d'une analyse préalable à l'analyse de l'activité, dans le domaine de la livraison de béton sur les chantiers du BTP. Éducation permanente, 165, 47-65.

Viennot, L. (1996). Raisonner en physique, la part du sens commun. Bruxelles: De Boeck-Westmael.

Villemin, G. (2005). Nombres : curiosités-théorie-usage, (en ligne : http://villemin.gerard.free.fr/index.html, site créé le 11/11/2005 et consulté le 9/12/2005).

Vincenti, W., G. (1990). What engineers know and how they know it. Baltimore: Johns Hopkins University Press.

Vincenti, W., G. (2001). The experimental assessment of engineering theory as a tool for design. Techne: Journal of the Society for Philosophy and Technology, 5(3), 31-39.

Vosniadou, S. \& Brewer, W.F. (1987). Theories of knowledge restructuring in developement. Review of educational research, 57, 51-67.

Vries (de), M. J. (2003). The nature of technical knowledge : extending empirically informated studies into what engineers know. Techne: Journal of the Society for Philosophy and Technology, 6(3), 1-21.

Vygotski, L. (1934/1997). Pensée et Langage. Paris: La dispute (3e édition, traduction : F. Sève).

Weill-Fassina, A., \& Pastré, P. (2004). Les compétences professionnelles et leur développement. In P. Falzon (Ed.), Ergonomie (pp. 213-231). Paris: PUF.

Weill-Fassina, A., Rabardel, P., \& Dubois, D. (Eds.) (1993) Les représentations pour l'action. Toulouse: Octarès.

\section{RÉSUMÉ}

Cet article a pour objectif de faire le point sur la place de la conceptualisation dans l'activité professionnelle et sur la notion de " concept pragmatique » (Samurçay, \& Pastré, 1995), qui occupe une place centrale en didactique professionnelle, comme composant de la compétence professionnelle, et comme organisateur de l'activité de travail. Après un bref historique sur l'introduction de cette notion, nous discutons de différentes approches qui ont abordé, directement ou indirectement, la conceptualisation pour l'action. Nous exposons ensuite en quoi la notion de concept pragmatique se différencie de celles de concepts scientifiques et techniques, d'une part, et de concept de concepts quotidiens, d'autre part. Nous indiquons quelle est la place spécifique que la notion de concept pragmatique occupe dans la théorie de didactique professionnelle, par rapport à celle de concept-en-acte (et de théorème-en-acte) que Vergnaud (1990) a développée initialement en didactique des mathématiques. Dans un dernier temps, nous discutons des apports que constitue l'intégration des questions de conceptualisation pour l'action à la conception d'outils d'aide, sous l'angle de la conception d'outils cognitifs opératifs.

MoTS-CLÉ

conceptualisation, développement des compétences professionnelles, concepts pragmatiques, outils cognitifs opératifs.

\section{RESUMEN}

\section{La conceptualización y el lugar de los conceptos pragmáticos en la}


actividad profesional y en el desarrollo de las competencias. Este artículo tiene como objetivo realizar una síntesis acerca de la conceptualización en la actividad profesional, así como sobre la noción de « concepto pragmático » (Samurçay \& Pastré, 1995) que ocupa un lugar central en la didáctica profesional, en tanto componente de la competencia profesional y como organizador de la actividad de trabajo. Luego de realizar una breve referencia sobre la introducción de esta noción, discutimos los diferentes enfoques que, directa o indirectamente, han abordado la conceptualización para la acción. Posteriormente desarrollamos las cuestiones que permiten diferenciar, por un lado, la noción de concepto pragmático de las de los conceptos científicos y técnicos y, por otro lado, de la de los conceptos cotidianos. Señalamos el lugar específico que ocupa la noción de concepto pragmático en la teoría de la didáctica profesional, con relación a la de concepto-enacto (y de teorema-en-acto) que, inicialmente, Vergnaud (1990) desarrolló en el marco de la didáctica de las matemáticas. Finalmente, discutimos los aportes que significan la integración de las cuestiones referidas a la conceptualización para la acción en el diseño de herramientas de ayuda, desde el enfoque del diseño de herramientas cognitivas operativas.

\section{PALABRAS-CLAVE}

Conceptualización, desarrollo de competencias profesionales, conceptos pragmáticos, herramientas cognitivas operativas.

Article reçu le 9 octobre 2006, accepté le 23 février 2007. 


\section{Annexes}

«1. Se souvenir du principe du «retour inverse de la lumière» afin d'imaginer le point de vue de l'objectif, en fonction du «cadre postérieur» que lui impose la fenêtre $24 \times 36 \mathrm{~mm}$ de l'image sur le film.

Le point de vue de l'objectif est inscrit dans une vaste pyramide isocèle à quatre faces (égales deux à deux, en regard) [...] cela signifie pyramide régulière, à base rectangulaire. Le sommet de cette pyramide est situé au centre optique de l'objectif et son axe est évidemment confondu avec l'axe optique, qui est perpendiculaire en son milieu au format $24 \times 36 \mathrm{~mm}$ de l'image projetée sur le film. La base de cette pyramide constitue le champ rectangulaire de l'image, situé dans le plan de mise au point. À cette pyramide en répond une autre, toute petite, située dans la chambre noire de l'appareil ; cette deuxième pyramide est également isocèle à quatre faces (égales deux à deux, en regard). Le sommet de cette petite pyramide est également le centre optique de l'objectif et son axe est également confondu avec l'axe optique. La base de cette petite pyramide constitue le format $24 \times 36$ mm de l'image projetée sur le film. Ces deux pyramides sont coaxiales et opposées par leur sommet commun (le centre optique de l'objectif). Elles sont également homothétiques, car leurs quatre arêtes suivent la première loi de Huygens [...] : loi de propagation rectiligne d'un rayon lumineux passant par le centre optique d'une lentille (à laquelle est assimilée l'objectif).

$[\ldots]$

2. Imaginer ensuite le point de vue du viseur lorsque la mise au point est réglée sur l'infini (la correction de parallaxe étant alors nulle).

Dans ce cas, le point de vue du viseur est également inscrit dans une vaste pyramide isocèle à quatre faces (égales deux à deux, en regard). Le sommet de cette pyramide est situé au «centre optique du viseur» [...]. La base de cette pyramide constitue le champ rectangulaire de l'image, situé à l'infini, dont le périmètre est confondu avec le cadre sélectionné dans le viseur. La hauteur de cette pyramide est confondue avec l'axe optique du viseur. Les axes optiques du viseur et de l'objectif sont parallèles et se coupent à l'infini, au centre du champ rectangulaire de l'image. Les points de vue de l'objectif et du viseur constituent deux pyramides égales, dont les bases sont confondues et situées à l'infini.

3. Imaginer maintenant le point de vue du viseur lorsque la mise au point n'est pas réglée sur l'infini, mais est au contraire proche (la correction de parallaxe étant alors effective) :

Dans ce cas, le point de vue du viseur est inscrit dans une pyramide scalène à quatre faces (toutes inégales) [...], contrairement au cas précédent, cette pyramide est irrégulière car son axe n'est pas perpendiculaire à sa base. Le sommet de cette pyramide demeure situé au «centre optique du viseur». La base de cette pyramide constitue le champ rectangulaire de l'image, situé dans le plan de mise au point, dont le périmètre est confondu avec le cadre sélectionné dans le viseur en raison de la correction de la parallaxe. L'erreur de parallaxe est parfaitement corrigée à l'intérieur de l'ensemble de ce cadre. L'erreur de parallaxe est parfaitement corrigée pour l'ensemble du cadre, mais seulement pour les objets situés dans le plan de la mise au point.

4. Imaginer enfin comment est perçue la réalité par les points de vue de l'objectif et du viseur, dans un plan différent du plan de mise au point, mais parallèle à celui-ci (par exemple, dans un plan rapproché). Ce plan coupe les deux points de vue selon deux rectangles se recouvrant partiellement. Pour ce plan, l'erreur de parallaxe n'est pas corrigée à l'intérieur du cadre sélectionné dans le viseur ».

Encadré 1 : Extrait d'une théorisation de la correction de la parallaxe dans le domaine de la photographie ${ }^{26}$.

26. Le texte est reproduit à l'identique. En gras, les éléments que nous soulignons. (D’après le site « La correction de la parallaxe ».) 


\section{— D., le 26 Avril 2005. Sujet du message: « télémètre et parallaxe»}

« Je suis l'heureux propriétaire d'un M6 mais je rencontre un petit souci sur bon nombre de mes images, en effet, je n'arrive pas à avoir un horizon (ou une ligne) droit(e) sur mes images et pourtant j'y suis très attentif lors de la prise de vue! (horizon droit au niveau du viseur mais horizon penché sur le négatif). Il me semblait que le viseur corrigeait les problèmes de parallaxe ! J'ai entendu parler de réglage du télémètre, ce réglage permet-il également de corriger ce défaut (s'il s'agit bien d'un défaut )! S'il y a une autre solution au problème, je suis preneur!

$[\ldots]$

Un exemple valant toujours mieux qu'un long discours, voici une image expliquant le problème rencontré :

[une photo est insérée dans le texte]

Image prise sur pied (mais sans utilisation de niveau à bulle), viseur vers le haut, donc optique se trouvant plus basse et pl gauche par rapport au viseur. Je suis sûr d'avoir bien vérifier que les lignes étaient bien droites dans le viseur! (surtout pour ce type d'image) [...]. »

—C. 27 Avril 2005. Sujet du message :

«Il est très difficile de réussir ce type de jeu avec la perspective avec un appareil à télémètre : le viseur n'est pas au même endroit que l'optique qui fait la photo, la perspective vue dans le viseur n'est donc pas celle de la photo. Dans ce cas-ci, le boîtier n'est pas parallèle au sujet et il y a contre-plongée. L'appareil doit être positionné de manière rigoureuse : bord bas du film parfaitement horizontal et parfaitement parallèle au sujet. À travers un viseur comme celui du « $\mathbf{M}$ », si l'axe du regard n'est pas sur l'axe du viseur, les informations vues peuvent avoir de la distorsion. Dans un reflex (de bonne qualité) l'image vue dans le viseur est projetée sur un dépoli, et correspond à celle qui sera projetée sur le plan film. Ne pas oublier que dans un appareil à télémètre avec correction de parallaxe, le centre de l'image visée est le centre de l'image projetée sur le plan film, mais pas depuis le même point de vue !

$[\ldots]$

Voici pourquoi la visée du M ne peut pas être parfaitement fidèle : schéma ci-dessous :

Cela n'enlève rien à la qualité du système : mise au point rapide et précise, légèreté et silence $[\ldots] »$.

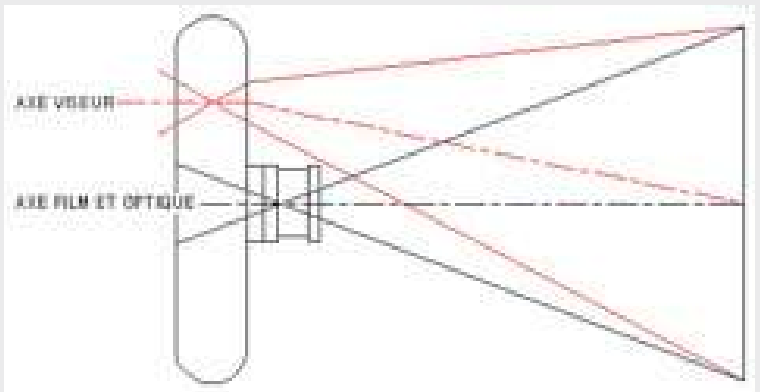

Encadré 2 : Exemple d'échanges sur une liste de diffusion en avril $2005^{27}$

On peut relever des échanges présentés (encadré 2) que D. connaît, au moins de façon globale, la notion de parallaxe (il l'indique dans le sujet de son message) et les problèmes posés ( $«$ il me semblait que le viseur corrigeait les problèmes de parallaxe »). Toutefois, les interventions de C. montrent que D. ne semble pas maîtriser les problèmes de parallaxe en intégrant les caractéristiques de son appareil. Les interventions de C. en soulignent deux aspects : 1) « le viseur n'est pas au même endroit que l'optique qui fait la photo, la perspective vue dans le viseur n'est donc pas celle de la photo »;2) «ne pas oublier que dans un appareil à télémètre avec correction de parallaxe, le centre de l'image visée est le centre de l'image projetée sur le plan film, mais pas depuis le même point de vue ».

Le schéma utilisé par C. pour expliquer le problème représente la différence entre ce que l'on voit dans le viseur (axe viseur) et ce qui sera photographié (axe film et optique), ce qui rend compte des propriétés de la parallaxe pertinentes pour l'action.

27. Le texte est reproduit à l'identique. En gras, les éléments que nous soulignons. 